\title{
Shape analysis of the neostriatum in subtypes of frontotemporal lobar degeneration: Neuroanatomically significant regional morphologic change
}

\author{
Jeffrey Chee Leong Looi ${ }^{\mathrm{a}, \mathrm{b}, *}$, Mark Walterfang ${ }^{\mathrm{c}}$, Martin Styner ${ }^{\mathrm{d}, \mathrm{e}}$, Marc Niethammer ${ }^{\mathrm{d}}$, Leif Anders Svensson ${ }^{\mathrm{f}}$, \\ Olof Lindberg $^{\mathrm{b}}$, Per Östberg ${ }^{\mathrm{b}, 1}$, Lisa Botes ${ }^{\mathrm{f}}$, Eva Örndahl ${ }^{\mathrm{f}, \mathrm{g}}$, Phyllis Chua ${ }^{\mathrm{h}}$, Dennis Velakoulis ${ }^{\mathrm{c}}$, \\ Lars-Olof Wahlund ${ }^{\mathrm{b}}$
}

${ }^{a}$ Research Centre for the Neurosciences of Ageing, Academic Unit of Psychological Medicine, School of Clinical Medicine, Australian National University Medical School, Canberra Hospital, Canberra, Australia

${ }^{\mathrm{b}}$ Karolinska Institute, Department of Neurobiology, Caring Sciences \& Society, Division of Clinical Geriatrics, Huddinge, Stockholm, Sweden

${ }^{\mathrm{c}}$ Melbourne Neuropsychiatry Centre, Royal Melbourne Hospital, and University of Melbourne, Australia

${ }^{\mathrm{d}}$ Department of Computer Science, University of North Carolina, Chapel Hill, NC, USA

e Department of Psychiatry, University of North Carolina, Chapel Hill, NC, USA

${ }^{\mathrm{f}}$ Hospital Physics \& Radiology, Karolinska University Hospital, Huddinge, Stockholm, Sweden

${ }^{\mathrm{g}}$ Karolinska Institute, Department of Clinical Science, Intervention and Technology, Division of Medical Imaging and Technology, Huddinge, Stockholm, Sweden

${ }^{\mathrm{h}}$ Alfred Psychiatry Research Centre, Monash University, Melbourne, Australia

\section{A R T I C L E I N F O}

\section{Article history:}

Received 14 May 2010

Received in revised form 25 August 2010

Accepted 26 September 2010

\section{Keywords:}

Striatum

Shape

Atrophy

Neuronatomy

Frontotemporal lobar degeneration

\begin{abstract}
A B S T R A C T
Frontostriatal circuit mediated cognitive dysfunction has been implicated in frontotemporal lobar degeneration (FTLD) and may differ across subtypes of FTLD. We manually segmented the neostriatum (caudate nucleus and putamen) in FTLD subtypes: behavioral variant frontotemporal dementia, FTD, $n=12$; semantic dementia, SD, $n=13$; and progressive non-fluent aphasia, PNFA, $n=9$ ); in comparison with controls $(n=27)$. Diagnoses were based on international consensus criteria. Manual bilateral segmentation of the caudate nucleus and putamen was conducted blind to diagnosis by a single analyst, on MRI scans using a standardized protocol. Intracranial volume was calculated via a stereological point counting technique and was used for normalizing the shape analysis. Segmented binaries were analyzed using the Spherical Harmonic (SPHARM) Shape Analysis tools (University of North Carolina) to perform comparisons between FTLD subtypes and controls for global shape difference, local significance maps and mean magnitude maps of shape displacement. Shape analysis revealed that there was significant shape difference between FTLD subtypes and controls, consistent with the predicted frontostriatal dysfunction and of significant magnitude, as measured by displacement maps. These differences were not significant for SD compared to controls; lesser for PNFA compared to controls; whilst FTD showed a more specific pattern in regions relaying fronto- and corticostriatal circuits. Shape analysis shows regional specificity of atrophy, manifest as shape deflation, with a differential between FTLD subtypes, compared to controls.
\end{abstract}

(c) 2010 Elsevier Ireland Ltd. All rights reserved.

\section{Introduction}

Frontotemporal lobar degeneration (FTLD) may be conceptualized as neurodegeneration of large-scale human brain networks (Seeley et al., 2009), with a predilection for specific subsystems, such as frontostriatal circuits, resulting in cognitive and behavioral decline (Looi et al., 2010). Frontostriatal circuits comprise a prefrontal region

\footnotetext{
* Corresponding author. Academic Unit of Psychological Medicine, Australian National University Medical School, Building 4, Level 2, Canberra Hospital, P.O. Box 11, Woden ACT 2605, Australia. Tel.: +6126244 3500; fax: +6126244 4964 .

E-mail address: Jeffrey.looi@anu.edu.au (J.C.L. Looi).

1 Present address: Department of Neuroscience, Uppsala University, Uppsala, Sweden.
}

which sends efferent pathways through the neostriatum (caudate nucleus, putamen) or nucleus accumbens, via the globus pallidus, onto the thalamus, and thence to specific prefrontal cortex (Alexander et al., 1986). Such circuits include motor loops originating in the frontal eye fields and supplementary motor cortex, as well as "cognitive loops" arising from dorsolateral prefrontal cortex, anterior cingulate cortex and orbitofrontal cortex (Alexander et al., 1986). Frontostriatal dysfunction may result in characteristic cognitive and behavioral syndromes (Cummings, 1993) in FTLD (Hodges and Patterson, 2007).

Dysfunction of frontostriatal circuits in FTLD may be reflected in structural change in components of that circuitry. Although FTLD has traditionally been conceptualized to arise from atrophy of frontal or temporal cortex, recent findings indicate that the neostriatum may be 
involved (Schroeter et al., 2007). In FTLD, cortical/subcortical atrophy and white matter changes (Zhang et al., 2009) may lead to disconnection of the neostriatum from frontostriatal circuits. Transsynaptic degeneration may be a useful model: in brief, it has been demonstrated that neuronal damage has anterograde (downstream) and retrograde (upstream) effects (Van Buren, 1963; Yucel and Gupta, 2008). Thus, trans-synaptic degeneration, either anterograde or retrograde (Van Buren, 1963; Yucel and Gupta, 2008), in the neural linkages of the neostriatum such as cortex, pallidum and thalamus, may result in reduction in the volume of the neostriatum. Long ago, severe atrophy of the caudate and putamen was noted post mortem in FTLD (von Braunmühl, 1930; von Bagh, 1946; Lüers and Spatz, 1957). Recent studies show neostriatal neuropathology may be significant in FTLD, especially in FTLD with TDP-43 positive intraneuronal inclusions (Davion et al., 2007; Leverenz et al., 2007) and FTD with motor neuron disease (Seelaar et al., 2007). Voxel-based morphometric studies of pathologically-confirmed FTLD have also shown reductions in striatal volume (Rabinovici et al., 2007); particularly in the FTD subtype (Whitwell et al., 2009); and evidence of associated circuit atrophy, present in early clinically diagnosed FTD (Seeley et al., 2008). Thus, neostriatal atrophy may be a hallmark of FTLD and its subtypes.

Our recent quantitative MRI studies of FTLD showed reductions in the volume of the caudate by up to $25 \%$ of control volume (FTD subtype) and by up to $13 \%$ of control volume in the putamen (PNFA subtype), in contrast to minimal volumetric change in Alzheimer's disease (AD) in comparison to controls (Looi et al., 2008b, 2009b). There was a gradation in atrophy consistent with the severity of theoretical frontostriatal dysfunction, with greatest caudate atrophy in the FTD subtype of FTLD, and uniform atrophy across all FTLD subtypes in the putamen.

Volumetric studies do not resolve regional morphologic change in the neostriatum; an ideal structure for morphological analysis due to the highly specific nature of regional interconnections. The caudate and putamen are divided into functional subregions based on afferents received from the frontal cortex and substantia nigra (Haber et al., 2000; Haber, 2003; Draganski et al., 2008). The caudate head and body receives connections on its lateral aspect from the dorsolateral prefrontal cortex, inferior orbitofrontal cortex, and posterior parietal cortex, whereas the tail receives input from the frontal eye fields. From its medial aspect, the caudate is connected to the anterior cingulate cortex (Alexander et al., 1986; Haber et al., 2000; Heimer and Van Hoesen, 2006; Utter and Basso, 2008). The putamen receives connections to its medial aspect from the motor cortex, and somatosensory cortex. The lateral putamen receives connections from the supplementary motor area. There are connections from the dorsolateral prefrontal cortex to the ventral aspect of the putamen (Haber et al., 2000; Haber, 2003). Regional changes in the shape of the caudate and putamen may be associated with dysfunction of specific circuits, resulting in clinical symptoms.

Spherical harmonic (SPHARM) shape analysis of the caudate and putamen has the potential to resolve localized areas of morphological change (Gerig and Styner, 2001; Styner et al., 2004, 2005, 2006). Levitt et al. (2004, 2009), Hwang et al. (2006), Choi et al. (2007) and Mamah et al. (2008) have previously applied shape analysis to the striatum in schizotypal personality disorder, bipolar disorder, obsessive-compulsive disorder, siblings of persons with schizophrenia, in aging (Koikkalainen et al., 2007) and Alzheimer's disease (Qiu et al., 2009) respectively.

We previously investigated whether FTLD (all subtypes combined) was associated with disturbed morphology of neostriatal structures using SPHARM (Looi et al., 2010); finding evidence of both general and regionally specific, clinically relevant, atrophy in FTLD, compared to AD and controls. Following on this, we hypothesized that FTLD subtypes differ in the putative degree of frontostriatal dysfunction (greater - FTD $>$ PNFA $>$ SD - lesser); were interested in whether subtypes showed specific patterns of neostriatal atrophy, and whether the relative contributions of subtypes to the neuroanatomical pattern of combined FTLD could be discerned.

Thus, based on our previous volumetric studies in neuropsychiatric disease and FTLD (Looi et al., 2008b, 2009a,b,c), and the morphologic change of the striatum shown in our shape analysis of FTLD, AD and controls (Looi et al., 2010); we hypothesized that the morphology of the caudate and putamen would differ between FTLD subtypes compared to controls, that is:

(1) Statistical group average shape of the caudate and putamen would differ between subtypes of FTLD and controls.

(2) Statistical group average shape of the caudate and putamen would differ between groups of FTLD subtypes and controls; based on the theoretical degree of frontostriatal circuit dysfunction (least shape change - SD $>$ PNFA $>$ FTD - greatest shape change);

(3) Statistical group average shape differences will be distributed in regions corresponding to the putative degree of involvement of frontostriatal circuits in disease groups.

\section{Methods}

\subsection{Participants}

Participants were recruited retrospectively from the Memory Clinic at the Karolinska University Hospital, Huddinge, Stockholm, Sweden and have been described in our previous papers (Looi et al., 2008b, 2009b, 2010). The study was approved by the local ethics committee.

Sixty-one subjects participated in the caudate study: 34 FTLD patients [12 frontotemporal dementia (FTD); 13 semantic dementia (SD); 9 progressive non-fluent aphasia (PNFA)]; and 27 in a control group [Table 1]. Two controls had strokes in the putamen and were excluded from this and subsequent studies, yielding 59 subjects for this study (thus, for the putamen, we had 34 with FTLD and 25 controls, with the other groups as above).

The clinical diagnosis was determined at a multidisciplinary consensus conference with physicians, neuropsychologists, speechlanguage pathologists and nurses. The medical examination included history from a close informant, as well as assessment of physical, neurological, and psychiatric status. Laboratory investigation of blood, CSF and urine (including vitamin B12, folic acid levels and thyroid function) was performed. Neuroradiologic examination consisted of magnetic resonance imaging (MRI) of the brain and single-photon emission computed tomography (SPECT) imaging of cerebral blood flow. Detailed EEG, neuropsychological and speech-language examinations were performed (see Looi et al., 2008b).

Table 1

Demographic features of patients and controls.

\begin{tabular}{lllll}
\hline & Control & FTD & SD & PNFA \\
\hline$N$ & 27 & 12 & 13 & 9 \\
Sex (M:F) & $7: 20$ & $3: 9$ & $4: 9$ & $3: 6$ \\
Age (years) & $61.1(53-78)$ & $59.5(42-72)$ & $63.8(52-77)$ & $64.9(57-78)$ \\
MMSE & $28.7(25-30)$ & $20.8(10-30)+$ & $22.9(5-29)+$ & $22.5(15-28) \#$ \\
Disease duration & - & 1.65 & 3.90 & 3.56 \\
$\quad$ (years) & & $(0.25-3.4)$ & $(1.3-7.7) \&$ & $(0.06-8.13)$ \\
\hline
\end{tabular}

$+, \#, \&$ Kruskal-Wallis test.

$+=P<0.01$ compared to controls.

$\#=P<0.05$ compared to controls.

$\&=P<0.01$ compared to FTD.

MMSE: mini-mental state examination score.

FTD: frontotemporal dementia.

SD: semantic dementia.

PNFA: progressive non-fluent aphasia.

$\mathrm{AD}$ : Alzheimer's disease. 


\subsubsection{FTLD}

Clinical criteria for the subtypes of FTLD were based on international consensus criteria (Neary et al., 1998). The subtypes included were: frontotemporal dementia (FTD), semantic dementia (SD) and progressive non-fluent aphasia (PNFA). We excluded patients with signs of cerebrovascular or systemic disorders. FTLD patients at different stages of the disease were included.

\subsubsection{Controls}

This group comprised individuals that were found, after careful assessment, not to fulfil criteria for FTLD, AD, or any other cognitive disorder, but who presented with subjective memory impairment at the memory clinic. Objective impairment was ruled out through comprehensive clinical, neuropsychological assessment and neuroimaging assessment. Neuropsychological impairment was defined as performance $\geq 1.5$ standard deviation unit below the mean on any cognitive test. Accordingly, controls had no objective cognitive impairment by definition. To further minimize the risk of including participants with neurodegenerative disease in very early stages as assessed by detailed clinical, neuropsychological and neuroimaging examination, only those participants that did not deteriorate on such assessments, over a minimum of two years follow-up were included.

\subsection{Imaging}

\subsubsection{Image acquisition}

T1-weighted MRI scans were acquired on a $1.5 \mathrm{~T}$ Siemens Magnetom Vision Plus scanner (Siemens Medical Systems, Erlangen, Germany). A three-dimensional magnetization-prepared rapid gradient echo (3D-MPRAGE) pulse sequence (TR $11.4 \mathrm{~ms}$; TE $4.4 \mathrm{~ms}$; TI $300 \mathrm{~ms}$; FA $10^{\circ}$; NEX 1) was used to obtain 72 contiguous coronal $2.5 \mathrm{~mm}$-slices with $512 \times 144$ matrix and $230-\mathrm{mm}$ field of view (FOV).

\subsubsection{Image analysis}

Volumetric analysis was performed using HERMES BMAP MorphyDisplay Scaled (Nuclear Diagnostics AB, Stockholm, Sweden). Preprocessing of imaging data was performed by: interpolation of the images to render them isotropic (voxels: $1 \mathrm{~mm}^{3}$ ); followed by alignment via automated rigid-body registration (AIR - Woods et al., 1998) to a customized local reference brain (in anterior commissureposterior commissure orientation); and adjusted by histogram signal intensity equalization to assist in standardizing intensity for more reliable manual segmentation.

All brain MRI scans were analyzed blindly to all clinical information (including diagnosis) by one experienced tracer. Based upon reference images, a standardized manual segmentation was performed, tracing the axial outline of the caudate serially through successive images. All portions of the caudate nucleus dorsal to the ventral aspect of the anterior commissure were included until the tail curved ventrally to border the lateral atrium of the lateral ventricles; here it was excluded from measurements. Similarly, the putamen was traced axially above the lowermost level of the anterior commissure where it could be identified as distinct to the caudate; demarcated by the external capsule laterally and the internal capsule medially, to its most dorsal aspect. The intra-rater reliability calculated as the intraclass correlation coefficient for the tracer was 0.98 for the caudate and 0.93 for the putamen for measurements separated by at least two weeks. Inter-rater reliability with another experienced tracer was assessed as 0.98 for the caudate and 0.87 for the putamen (Looi et al., 2008a,b, 2009b).

\subsection{Shape analysis}

Shape analysis was undertaken in a semi-automated fashion using the University of North Carolina shape analysis toolkit (http://www. nitrc.org/projects/spharm-pdm/); a detailed description of the meth- odology is available in (Styner et al., 2006; Levitt et al., 2009 - See Appendix for detailed summary). Segmented 3D binaries are initially processed to ensure interior holes are filled, followed by morphological closing and minimal smoothing. These are then subjected to spherical harmonic shape description (SPHARM), whereby boundary surfaces of each shape are mapped onto the surface of a sphere and the surface coordinates were represented through their spherical harmonic coefficients (Brechbuhler et al., 1995). The correspondence between surfaces is established by parameter-based rotation, itself based on first-order expansion of the spherical harmonics. The surfaces are uniformly sampled into a set of 1002 surface points and aligned to a study-averaged template for each structure (left and right caudate and putamen) using rigid-body Procrustes alignment (Bookstein, 1997), and, since scale information is more easily interpreted in the original unit space, the study-averaged template is normalized for head size using intracranial volume (ICV) via dividing scale information by a scaling factor, $f_{i}$, where $f_{i}=\left(\text { Mean }(\mathrm{ICV}) / \mathrm{ICV}_{\mathrm{i}}\right)^{1 / 3}$ (Styner et al., 2007).

\subsection{Shape statistical analyses}

We compute non-parametric statistical tests that compare the local surface coordinates for group mean differences at the 1002 surface locations (Styner et al., 2006, 2007; Levitt et al., 2009). A local group difference metric between groups of surface coordinates is derived from the Hotelling $T^{2}$ two sample metric (Styner et al., 2007). As the shape analysis involves computing 1002 hypothesis tests, one per surface location, a correction for multiple testing is necessary, as an uncorrected analysis would be overly optimistic. The shape analysis uses permutation tests of the Hotelling $T^{2}$ metric for the computation of the raw uncorrected $P$-values and false discovery rate (FDR) (Genovese et al., 2002) for multiple comparison correction.

Shape statistical analysis significance maps showing local statistical $P$-values, raw and corrected for FDR, are generated. A global shape difference is computed, summarizing average group differences across the surface. Statistical shape analysis also provides visualizations of the local effect size via mean difference magnitude displacement maps, which display the magnitude of surface change (deflation or inflation) in mm between corresponding points on the mean surfaces of group 1 and group 2. The color scale of the magnitude displacement visualizations varies due to the different degrees of deflation in individual comparisons (e.g. FTLD versus controls), with greater degrees of deflation requiring some compression of the color scale.

\section{Results}

\subsection{Shape analyses}

We applied the shape analysis method to the segmented caudate and putamen for the entire dataset. All results were scaled for total intracranial volume. The results presented are based upon FDR corrected $P$-value maps, together with corresponding local displacement maps. We have presented volumetric data in previous papers (Looi et al., 2008b, 2009b).

\subsection{Global shape measures (Table 2)}

The raw global shape measure describes the average Hotelling $T^{2}$ group difference metric across the whole surface, the single $P$-value is calculated by non-parametric permutation. On raw global shape measures, the bilateral neostriatum is significantly different in shape in FTD versus controls and in PNFA versus controls [Table 2]. For SD compared to controls, there is a global shape difference for only the left caudate nucleus. Our global between-subtype comparisons yielded few statistically significant results: shape change in the left 
Table 2

Global shape analysis $P$-values.

\begin{tabular}{|c|c|c|c|c|c|c|}
\hline \multirow[b]{2}{*}{ Group 1} & \multicolumn{6}{|c|}{ Comparison and $P$-values } \\
\hline & FTD & SD & PNFA & FTD & FTD & PNFA \\
\hline Group 2 & C & C & C & PNFA & SD & SD \\
\hline \multicolumn{7}{|l|}{ Structure } \\
\hline L Caud & $0.00005^{*}$ & $0.00005^{*}$ & $0.00005^{*}$ & 0.5539 & $0.01205^{*}$ & $0.0146^{*}$ \\
\hline R Caud & $0.00005^{*}$ & 0.09825 & $0.0035^{*}$ & 0.35115 & 0.02375 & 0.1574 \\
\hline L Put & $0.00215^{*}$ & 0.0648 & $0.0023^{*}$ & 0.5329 & 0.0734 & 0.2101 \\
\hline R Put & $0.00215^{*}$ & 0.0648 & $0.0023^{*}$ & 0.19095 & 0.13185 & $0.0209^{*}$ \\
\hline
\end{tabular}

Caud: caudate.

Put: putamen.

FTD: frontotemporal dementia.

SD: semantic dementia.

PNFA: progressive non-fluent aphasia.

C: controls.

* $P<0.05$.

caudate being significantly different between: FTD and SD; SD and PNFA; and in the right putamen between SD and PNFA [Table 2]. However, the regional significance and mean displacement maps for all the between-subtype comparisons were unremarkable, indicating these changes, though significant, were otherwise non-specific in terms of regional shape change. As the sample sizes of the betweensubtype comparisons were small, the regionally significant differences would be unlikely to pass SPHARM FDR correction.

\subsection{Shape measures: Significance maps and displacement maps}

For ease of reference, we present the data by structure (caudate or putamen), including the $P$-value shape significance maps and mean difference displacement maps for significant results.

\subsubsection{P-value significance maps}

The $P$-value color significance scale is identical for all images, and warmer colors refer to smaller $P$-values less than 0.05 , with the blue color corresponding to $P$-values above 0.05 .

\subsubsection{Mean difference magnitude displacement maps}

Note that the displacement color scale is unique for each image, and corresponds to the millimetres of deflation of the surface in that region; with warmer colors (such as red) corresponding to greater degrees of deflation, and cooler colors (such as green) lesser degrees of deflation. We have displayed volumes of deflation as positive values, such that the values represent millimetres of deflation of the overall larger structure compared to the smaller structure. This requires assignment of the "nominal" larger structure for comparison, based upon our previous volumetric studies for both caudate and putamen, where we found FTLD had smaller volumes than controls (Looi et al., 2008b, 2009b). Thus, for all comparisons, the group assigned as nominally larger, was the control group. Thus, the values of deflation describe the degree of deflation of the FTLD subtype compared to controls.

Additionally, the displacement direction was only in one direction in all analyses, without bi-directional shape changes. This was confirmed by using the visualizations of signed directional changes, which revealed unidirectional changes in all comparisons. Therefore, we used a unidirectional (non-signed) scale display.

As the between-subtype comparisons of FTD, SD and PNFA (e.g., FTD vs. SD) showed no significant regional differences, those maps are omitted from the article.

\subsubsection{Caudate maps [Figs. 1-3]}

FTD vs. Controls right caudate local P-value significance map [Fig. 1a]

FTD vs. Controls right caudate mean difference magnitude displacement map [Fig. 1b]
FTD vs. Controls left caudate local P-value significance map [Fig. 1c]

FTD vs. Controls left caudate mean difference magnitude displacement map [Fig. 1d]

For FTD compared to controls [Fig. 1a,c] there is widespread significance for difference in shape in the bilateral caudate. The displacement maps showed relatively more posterior shape deflation in the bilateral caudate of the order of $2 \mathrm{~mm}$ [Fig. 1b,d]

$S D$ vs. Controls right caudate local P-value significance map [Fig. 2a]

$S D$ vs. Controls right caudate mean difference magnitude displacement map [Fig. 2b]

$S D$ vs. Controls left caudate local P-value significance map [Fig. 2c]

$S D$ vs. Controls left caudate mean difference magnitude displacement map [Fig. 2d]

Comparing SD to controls, the local caudate significance maps show small regional differences, confined to the left caudate [Fig. 2c]. The displacement maps concurred with the significance maps, showing very mild shape deflation in SD in the left caudate approximately $1 \mathrm{~mm}$ [Fig. $2 \mathrm{~d}$ ].

PNFA vs. Controls right caudate local P-value significance map [Fig. 3a]

PNFA vs. Controls right caudate mean difference magnitude displacement map [Fig. 3b]

PNFA vs. Controls left caudate local P-value significance map [Fig. 3c]

PNFA vs. Controls left caudate mean difference magnitude displacement map [Fig. 3d]

Comparing PNFA to controls, the differences are again lateralized to the left [Fig. 3a,b], with approximately $3 \mathrm{~mm}$ of deflation of the caudate.

\subsubsection{Putamen maps [Figs. 4-6]}

FTD vs. Controls right putamen local P-value significance map [Fig. 4a] FTD vs. Controls right putamen mean difference magnitude displacement map [Fig. 4b]

FTD vs. Controls left putamen local P-value significance map [Fig. 4c]

FTD vs. Controls left putamen mean difference magnitude displacement map [Fig. 4d]

For FTD compared to controls [Fig. 4a-d], there is limited atrophy in the putamen, indicating a lesser involvement of the putamen. The putamen showed approximately $1 \mathrm{~mm}$, of generalized shape deflation between FTD and controls [Fig. 4b,d].

$S D$ vs. Controls right putamen local P-value significance map [Fig. 5a]

$S D$ vs. Controls right putamen mean difference magnitude displacement map [Fig. 5b]

$S D$ vs. Controls left putamen local P-value significance map [Fig. 5c]

$S D$ vs. Controls left putamen mean difference magnitude displacement map [Fig. 5d]

For the putamen in SD compared to controls, there were no significant differences in the regional or displacement maps [Fig. 5ad].

PNFA vs. Controls right putamen local P-value significance map [Fig. 6a] $P N F A$ vs. Controls right putamen mean difference magnitude displacement map [Fig. 6b]

PNFA vs. Controls left putamen local P-value significance map [Fig. 6c] PNFA vs. Controls left putamen mean difference magnitude displacement map [Fig. 6d]

Interestingly, the pattern of putaminal atrophy in PNFA is very similar to that in FTD [Fig. 6a-d], suggesting homogeneity in the pattern of neostriatal neurodegeneration of FTD and PNFA.

\section{Discussion}

To our knowledge, this is the first study quantifying neostriatal shape differences between FTLD subtypes compared to controls. The analysis reveals both general and specific shape change of neuroanatomical significance. 
a

Right Caudate - Significance Maps

Right Caudate - Lateral View

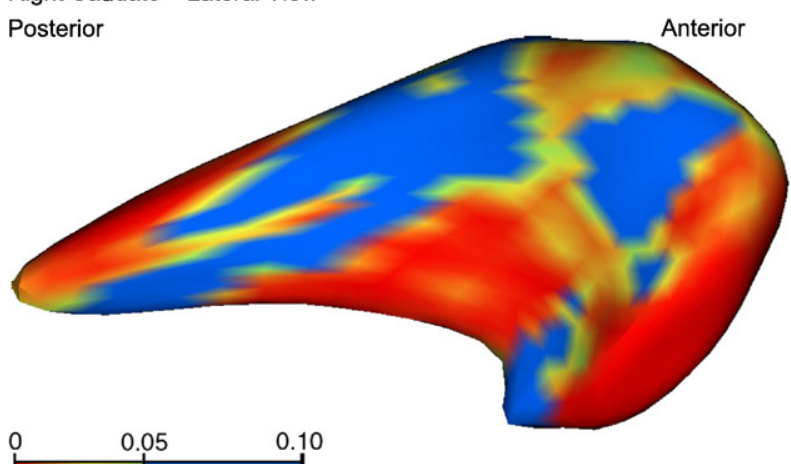

Right Caudate - Medial View Anterior

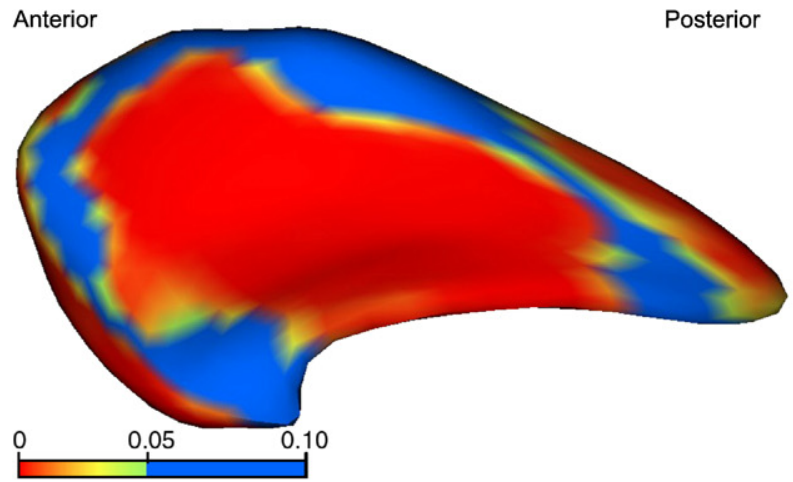

C

Left Caudate - Significance Maps

Left Caudate - Lateral View

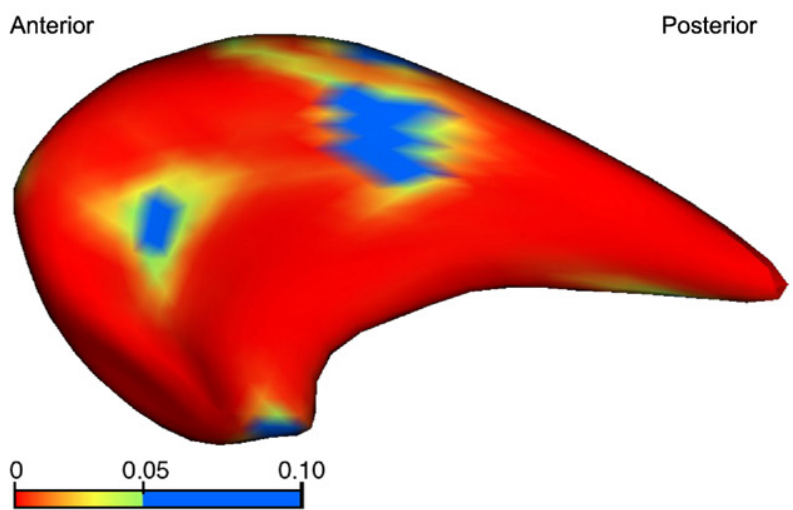

Left Caudate - Medial View

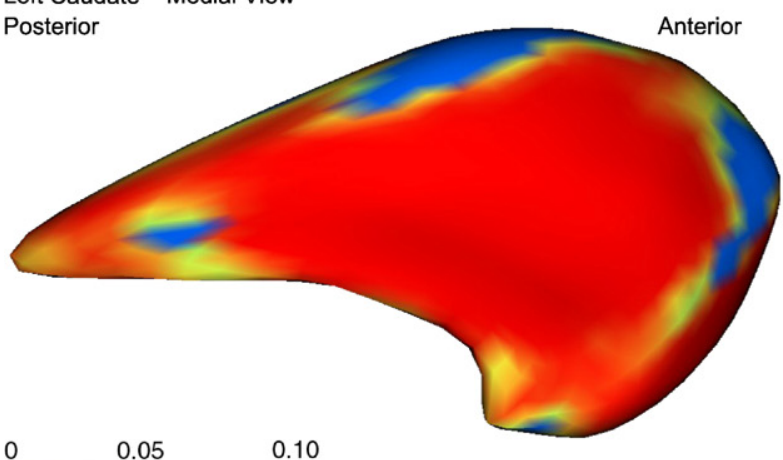

b

Right Caudate - Distance Maps

Right Caudate - Lateral view

Posterior

Anterior

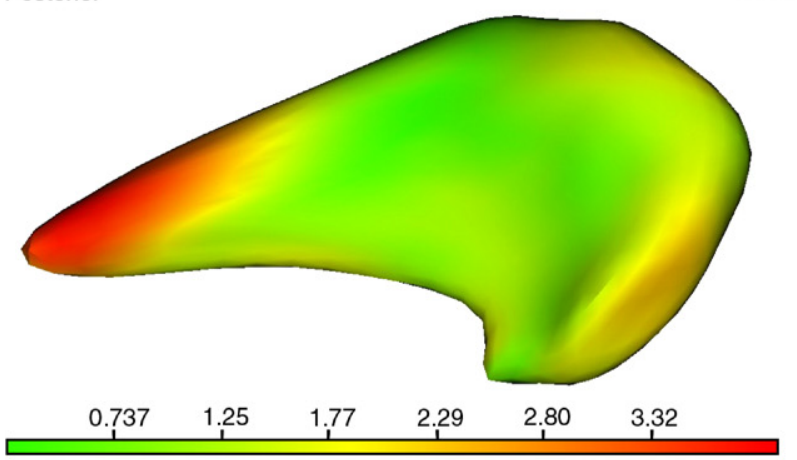

Right Caudate - Medial view

Anterior

Posterior

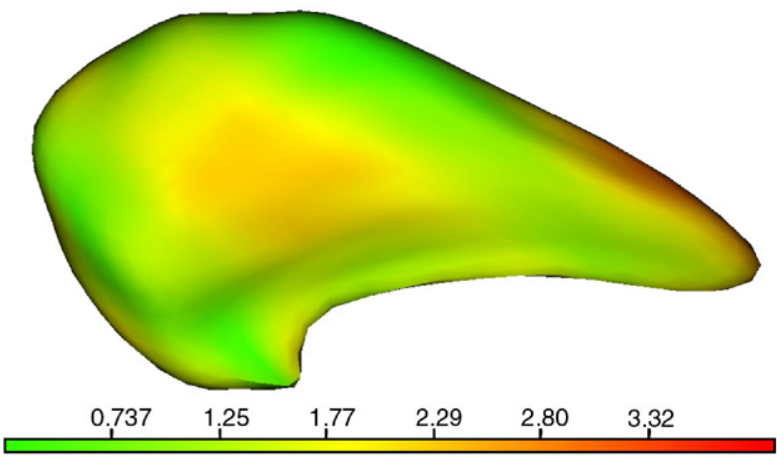

d

Left Caudate - Distance Maps

Left Caudate - Lateral View

Anterior

Posterior

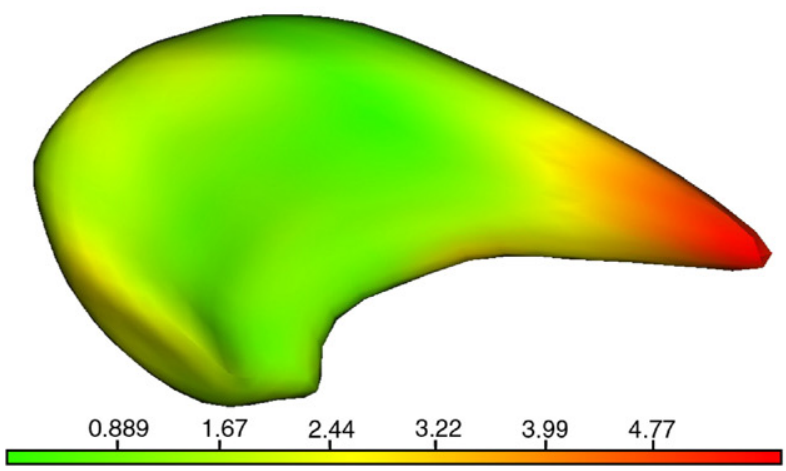

Left Caudate - Medial View

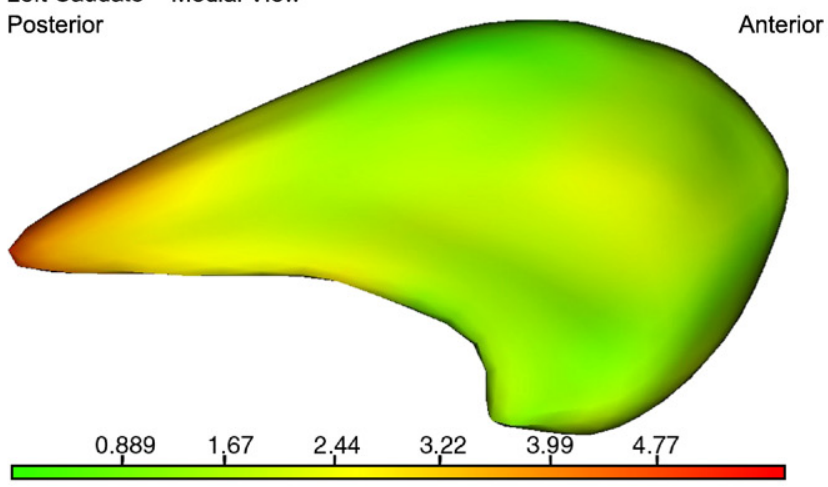

Fig. 1. FTD versus controls. 
a

Right Caudate-Significance Maps

Right Caudate - Lateral View

Posterior
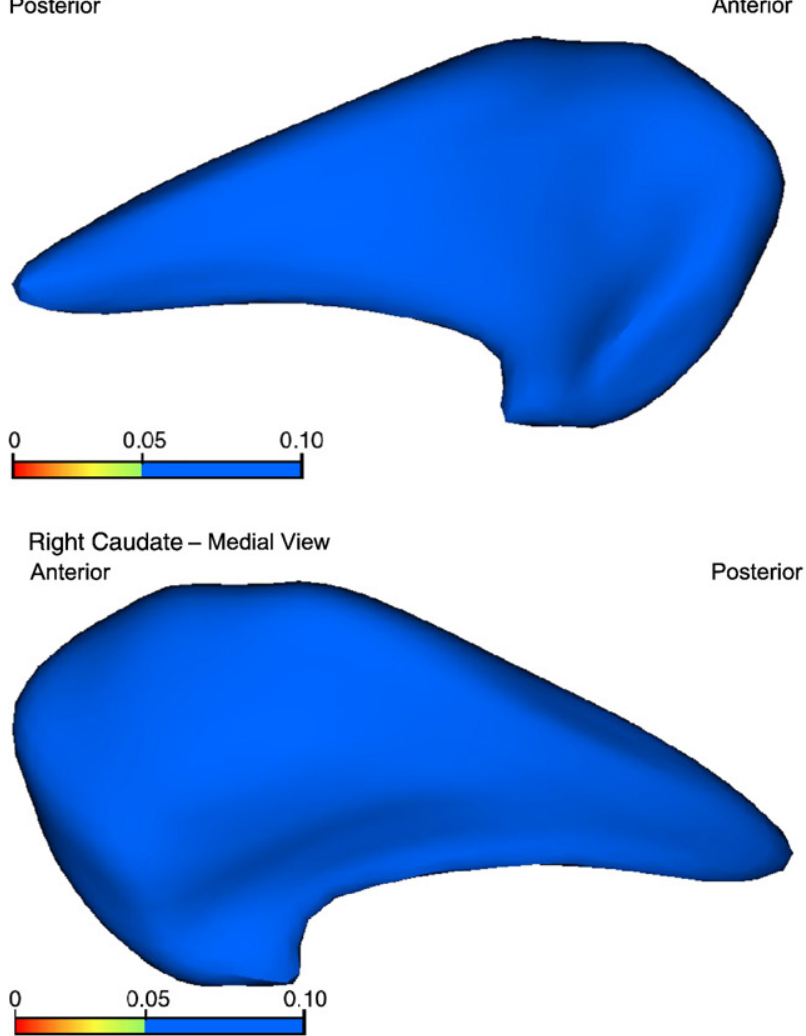

\section{C}

Left Caudate - Significance Maps

Left Caudate - Lateral View

Anterior

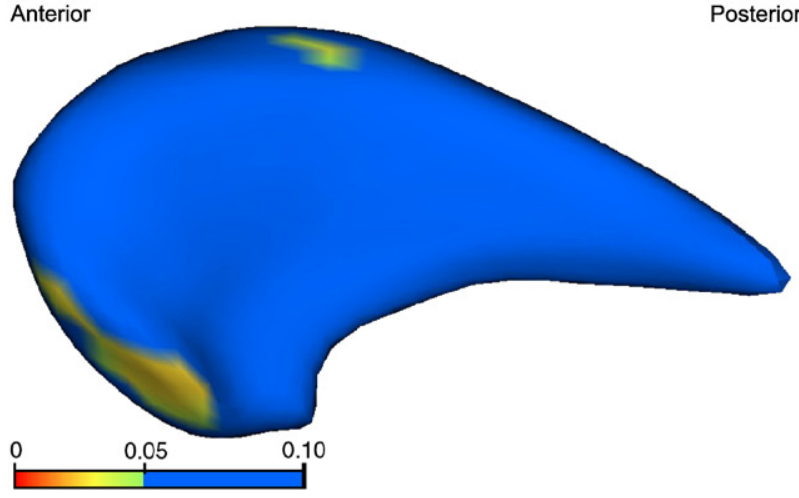

Left Caudate - Medial View

Posterior

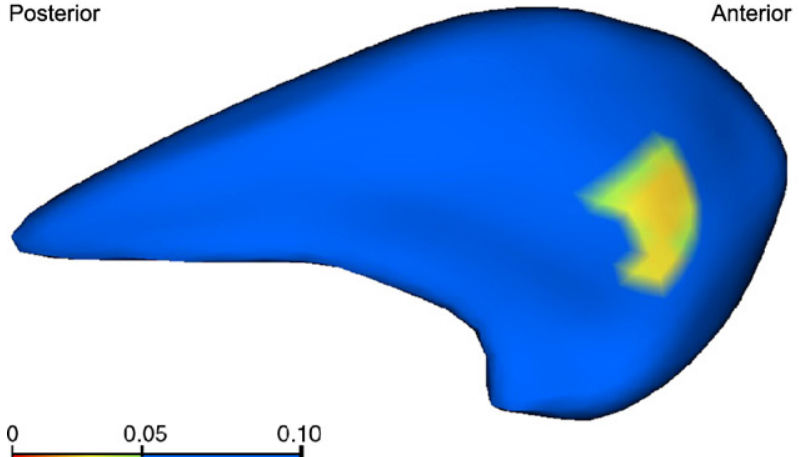

b

Right Caudate - Distance Maps

Right Caudate - Lateral view

Posterior Anterior

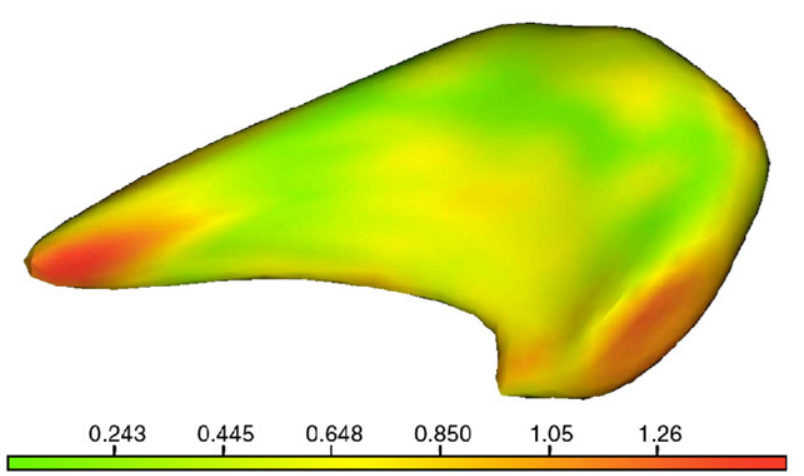

Right Caudate - Medial view

Anterior Posterior

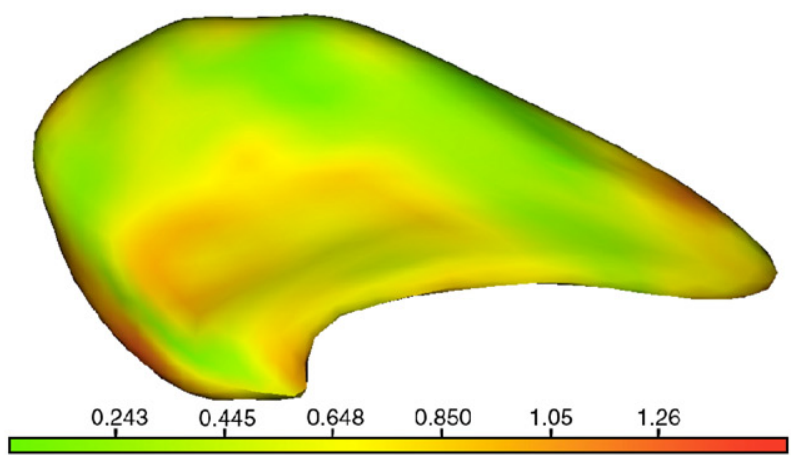

d

Left Caudate - Distance Maps

Left Caudate - Lateral View

Anterior

Posterior

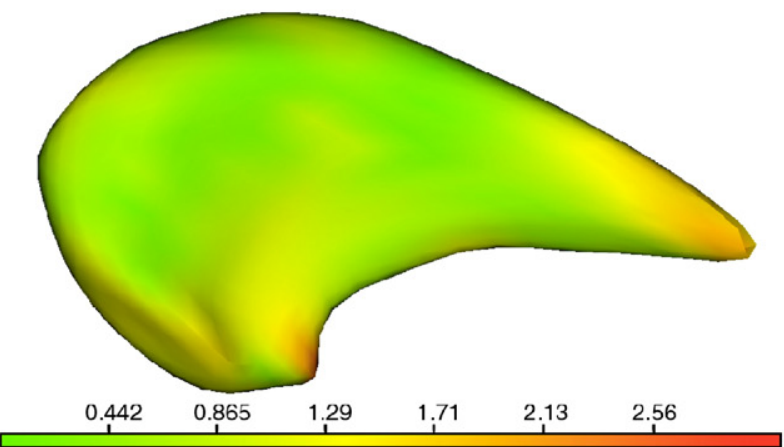

Left Caudate - Medial View

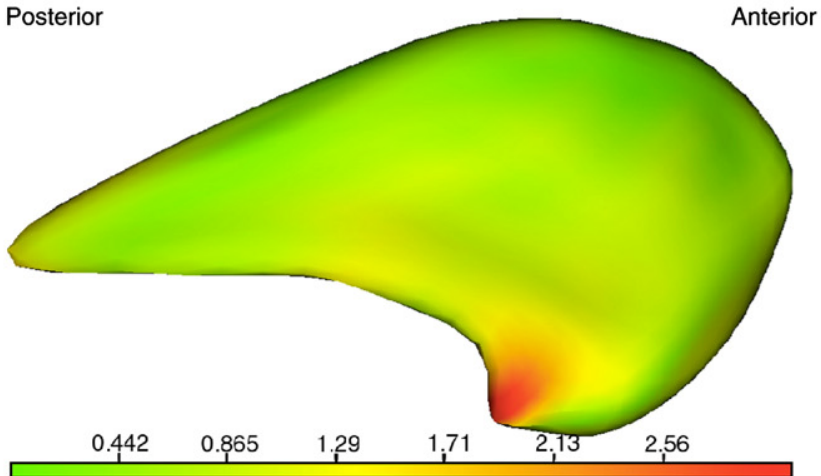

Fig. 2. SD versus controls. 
a

Right Caudate - Significance Maps

Right Caudate - Lateral View

Posterior
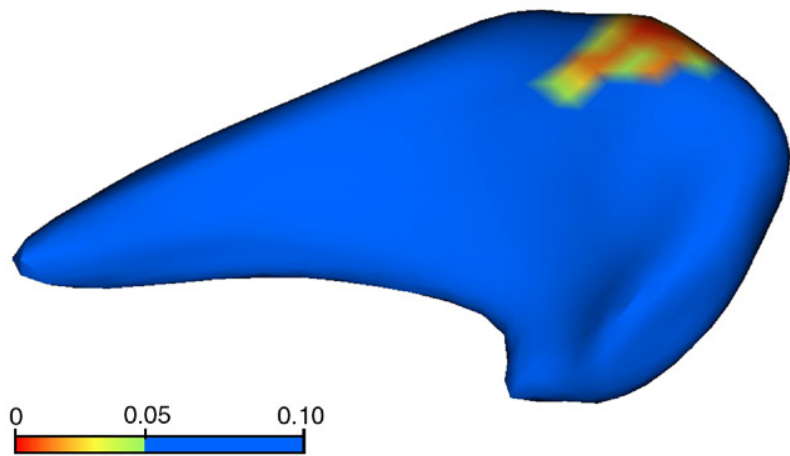

Right Caudate - Medial View

Anterior

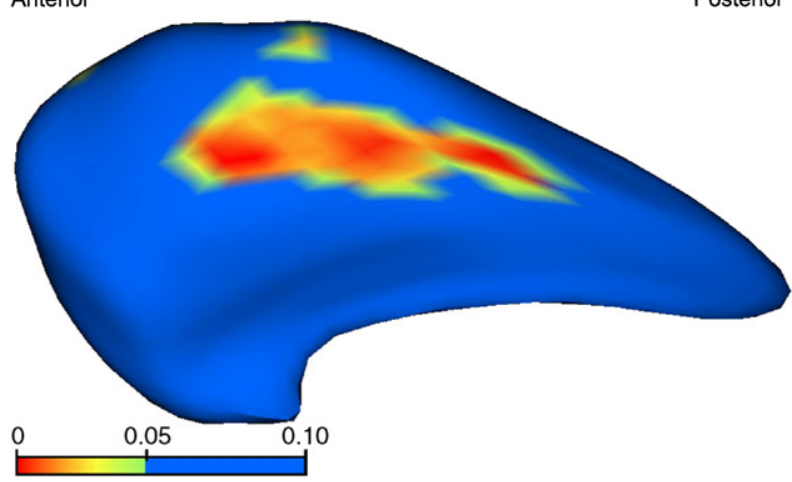

C

Left Caudate - Significance Maps

Left Caudate - Lateral View

Anterior

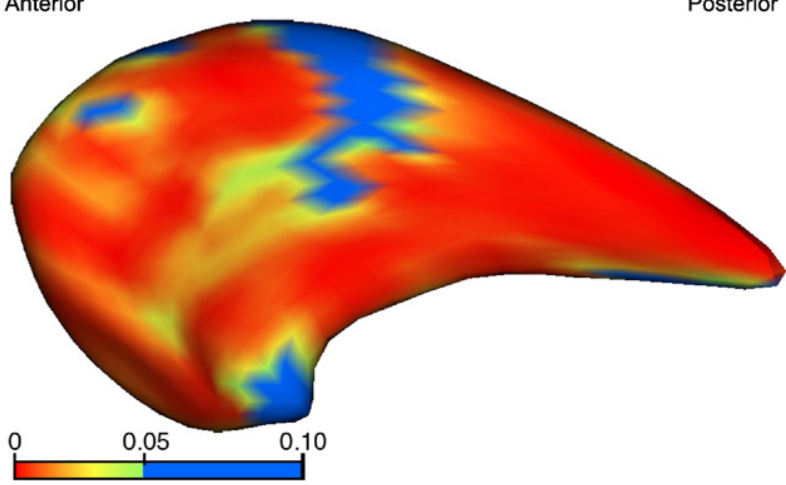

Left Caudate - Medial View Posterior

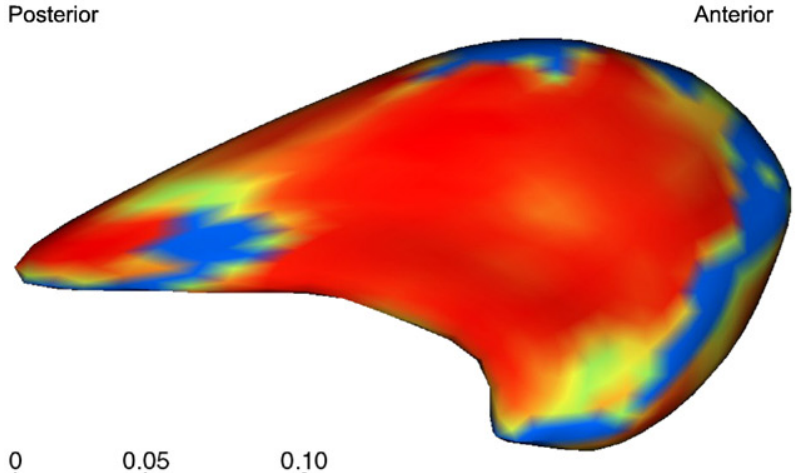

\section{b}

Right Caudate - Distance Maps

Right Caudate - Lateral view

Posterior

Anterior

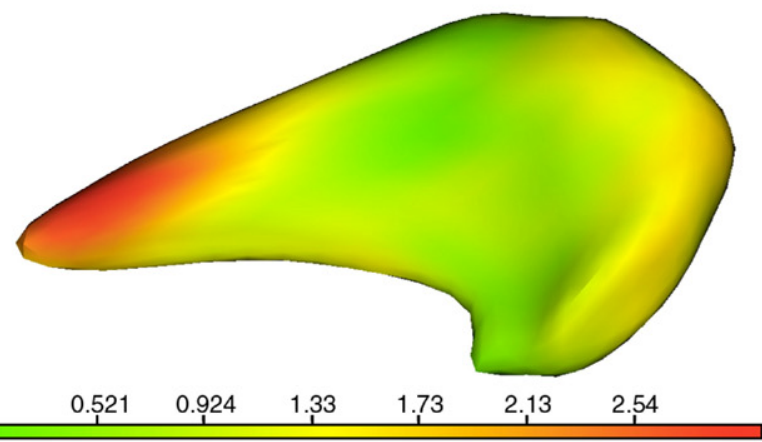

Right Caudate - Medial View

Anterior

Posterior

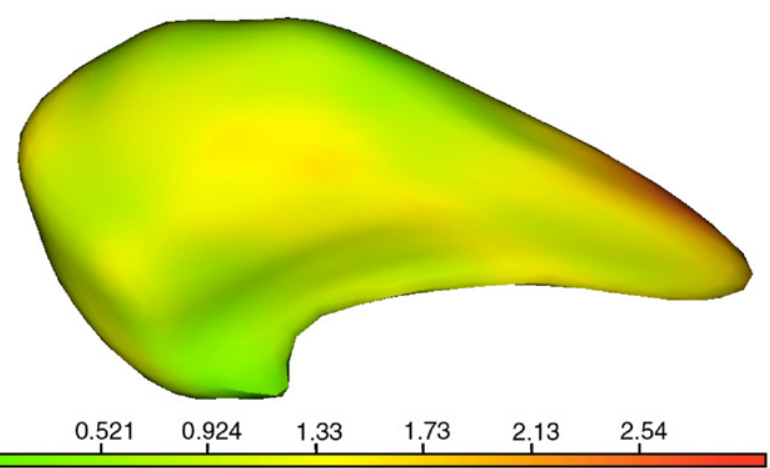

d

Left Caudate - Distance Maps

Left Caudate - Lateral View

Anterior

Posterior

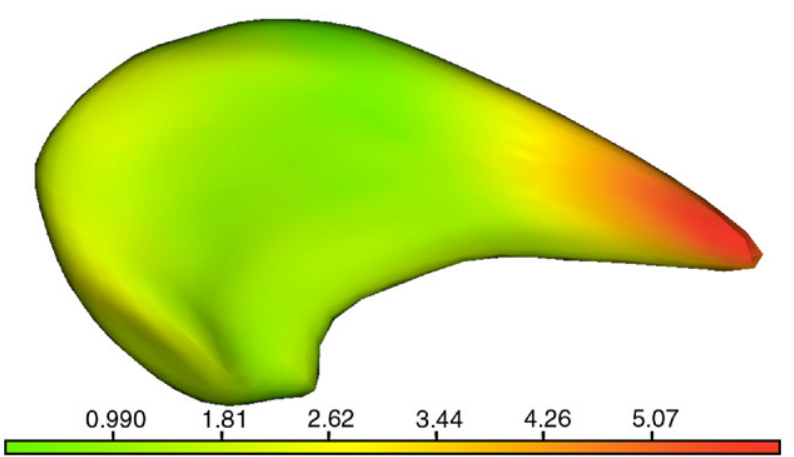

Left Caudate - Medial View

Posterior

Anterior

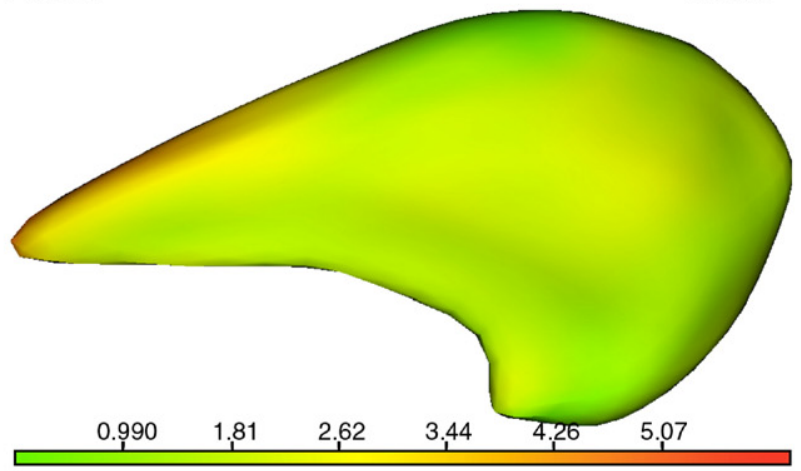

Fig. 3. PNFA versus controls. 
a

Right Putamen - Significance Maps

Right Putamen - Lateral View

Posterior

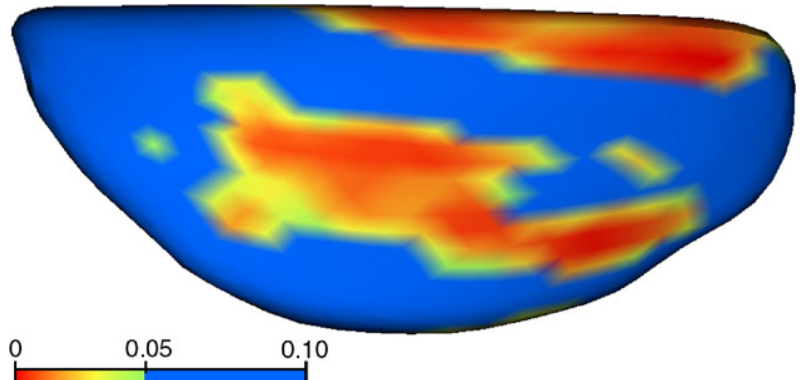

Right Putamen - Medial View

Anterior

Posterior

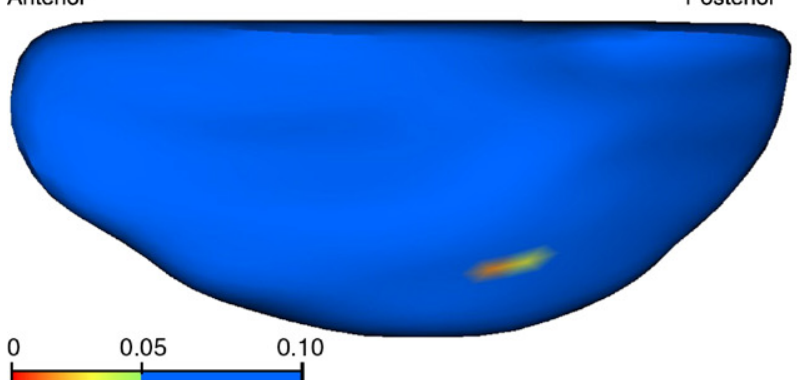

C

Left Putamen - Significance Maps

Left Putamen - Lateral View

Anterior

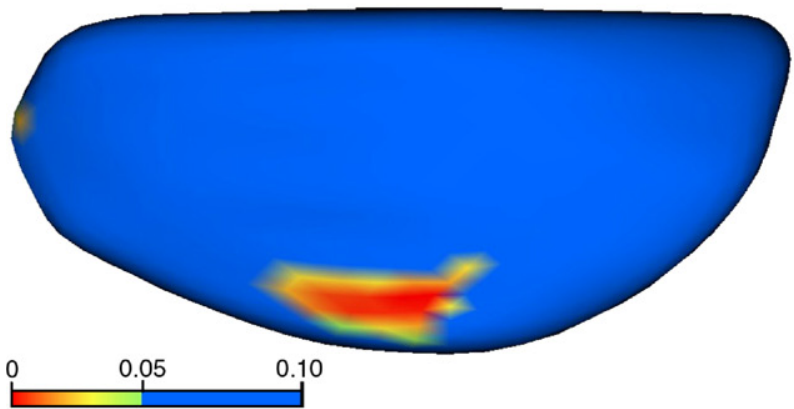

Left Putamen - Medial View Posterior

Anterior

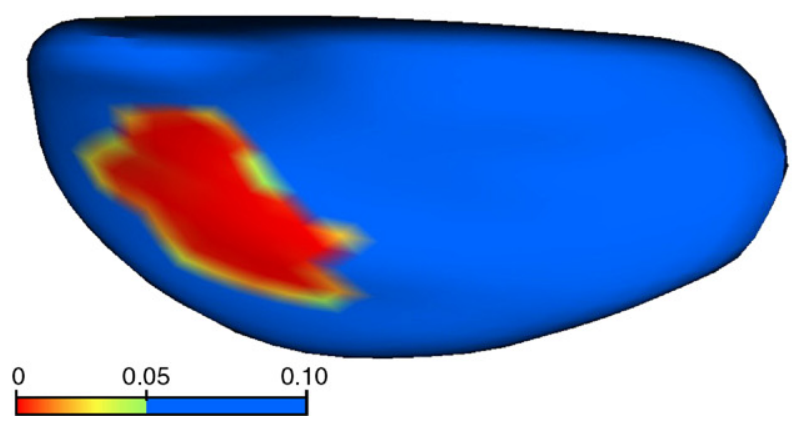

b

Right Putamen - Distance Maps

Right Putamen - Lateral View

Posterior

Anterior

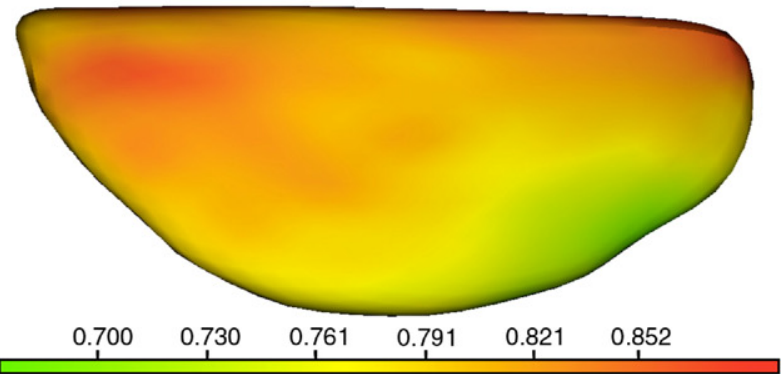

Right Putamen - Medial View

Anterior

Posterior

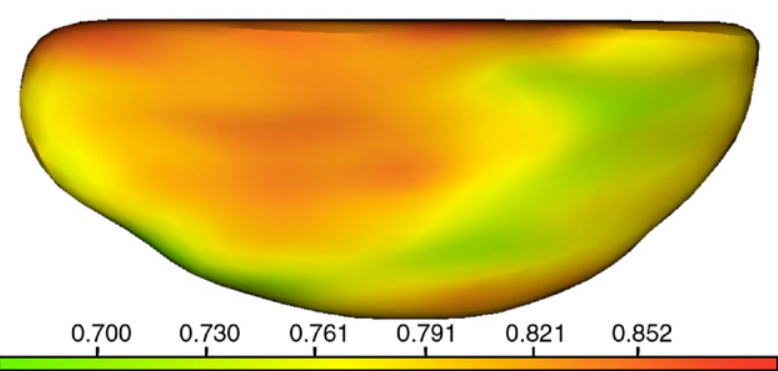

d

Left Putamen - Distance Maps

Left Putamen - Lateral View

Anterior

Posterior

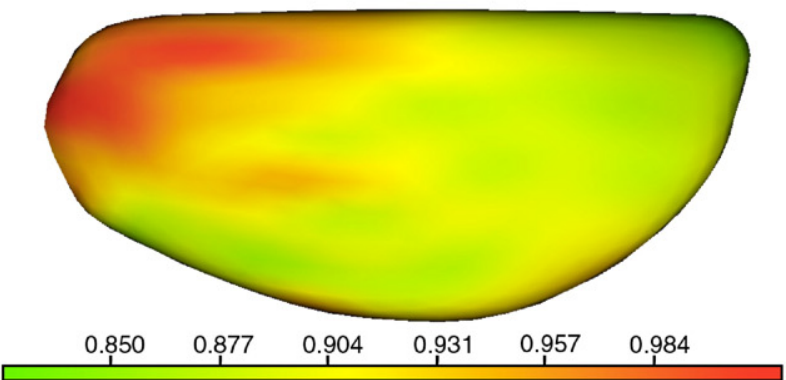

Left Putamen - Medial View

Posterior Anterior

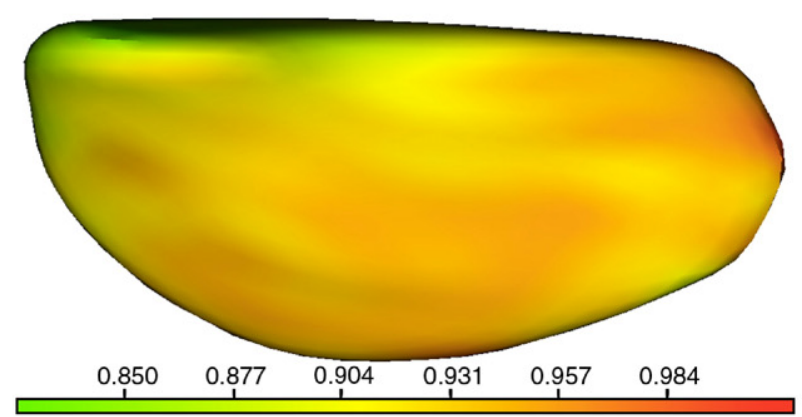

Fig. 4. FTD versus controls.

\subsection{Hypothesis 1: Global shape differences}

The finding of greater global shape deflation in the FTD and PNFA subtypes of FTLD, in comparison to controls, is supportive of a potential neostriatal structural basis for frontostriatal dysfunction in these subtypes. In contrast, in SD there is limited evidence of neostriatal deflation, and the left-lateralized difference of SD with controls may reflect corticostriatal disconnection 
a

Right Putamen - Significance Maps

Right Putamen - Lateral View

Posterior

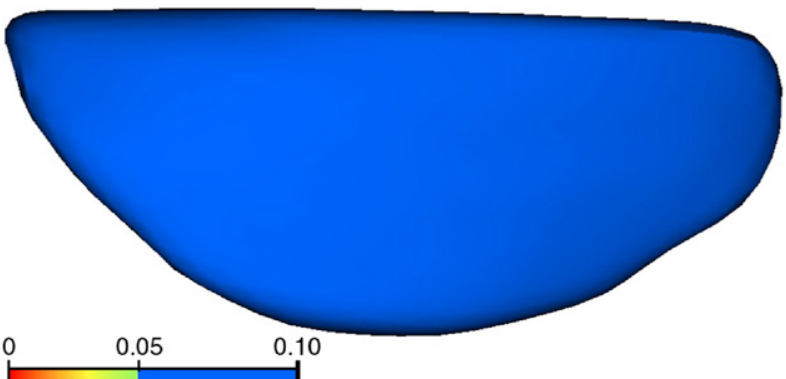

Right Putamen - Medial View

Anterior

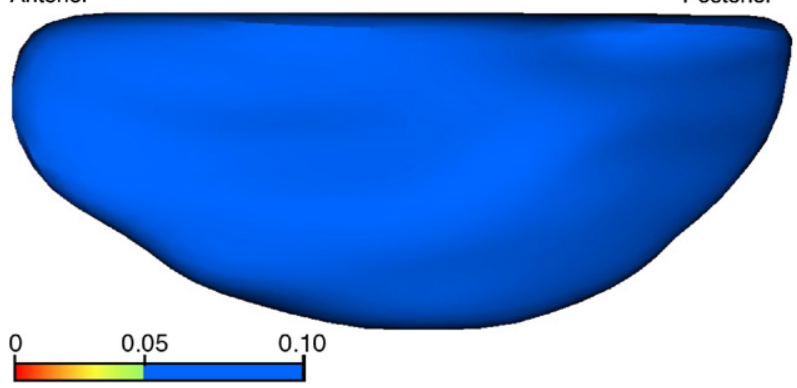

\section{C}

Left Putamen - Significance Maps

Left Putamen - Lateral View

Anterior

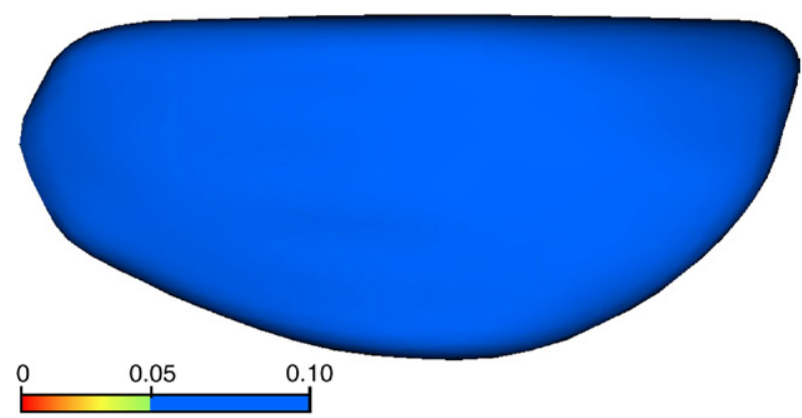

Left Putamen - Medial View

Posterior

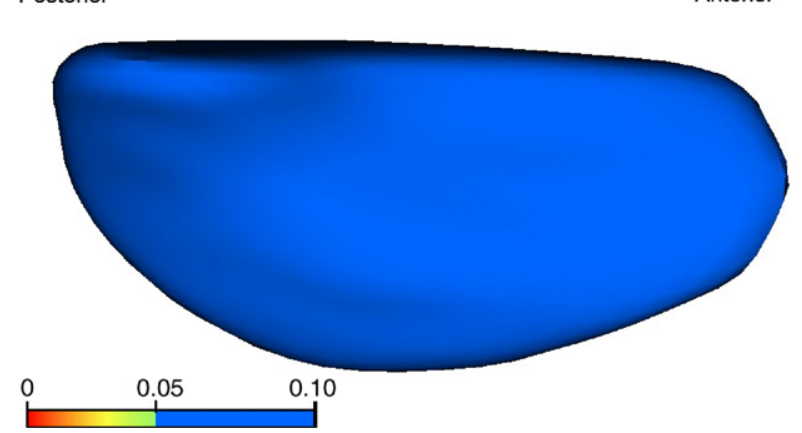

b

Right Putamen - Distance Maps

Right Putamen - Lateral View

Posterior

Anterior

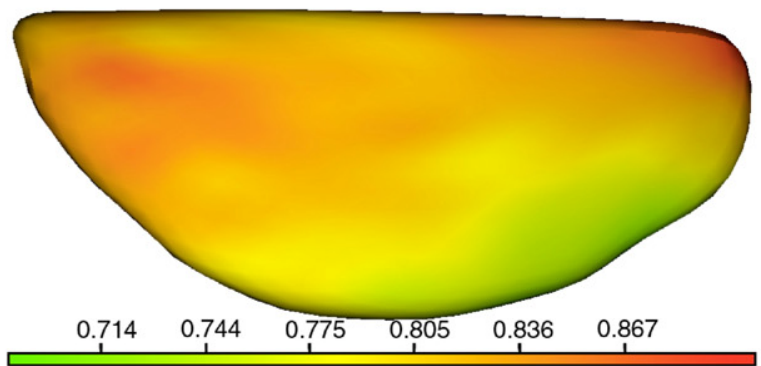

Right Putamen - Medial View

Anterior

Posterior

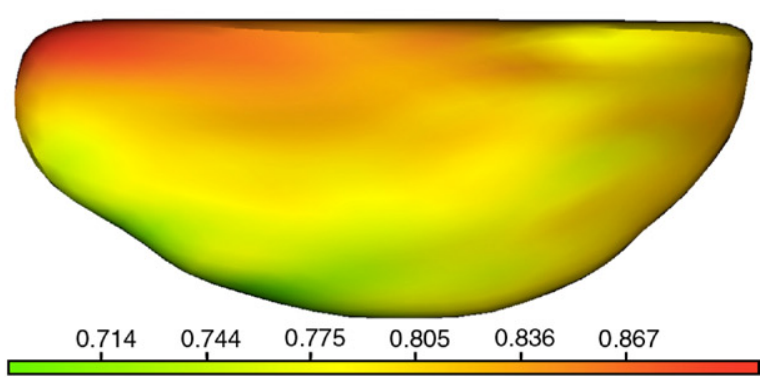

d

Left Putamen - Distance Maps

Left Putamen - Lateral View

Anterior

Posterior

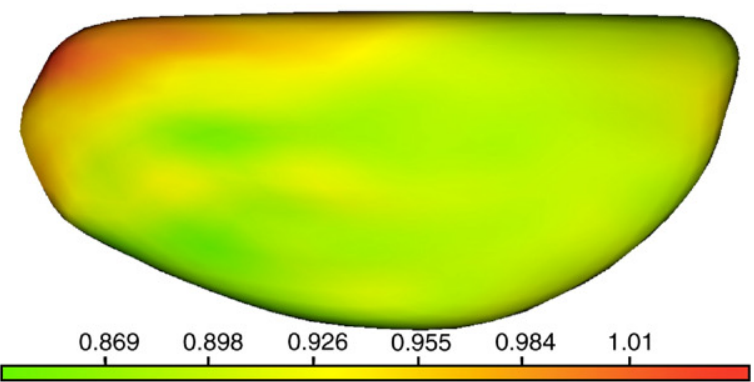

Left Putamen - Medial View

Posterior

Anterior

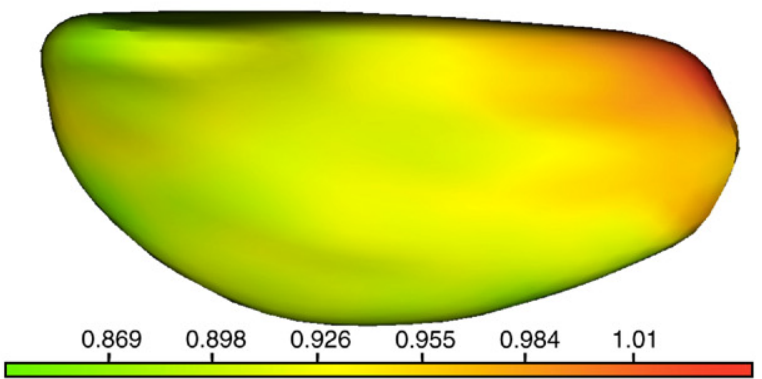

Fig. 5. SD versus controls.

related to the temporal lobe language regions. However, the lack of between FTLD subgroup differences may reflect the relative heterogeneity of these clinical subtypes, and the limitations of SPHARM with small sample comparisons when not including controls.

\subsection{Hypothesis 2 and 3: Patterns of atrophy caudate}

\subsubsection{FTD}

The pattern of atrophy in FTD is consistent with considerable neurodegeneration. That the majority of the shape differences are 
a

Right Putamen - Significance Maps

Right Putamen - Lateral View

Posterior

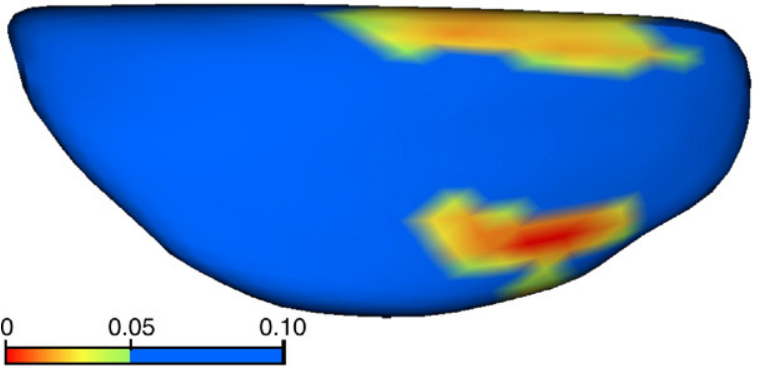

Right Putamen - Medial View Anterior Posterior

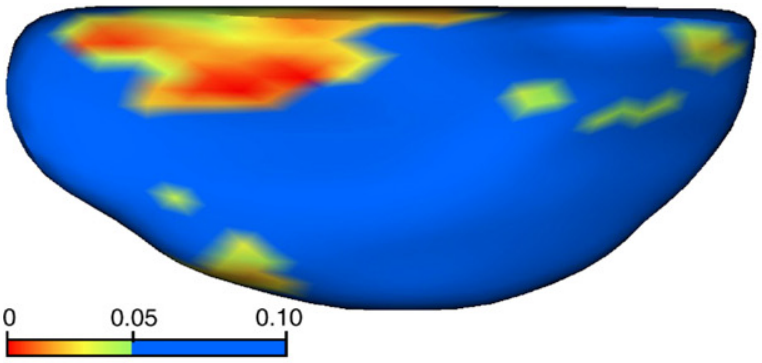

\section{C}

Left Putamen - Significance Maps

Left Putamen - Lateral View

Anterior

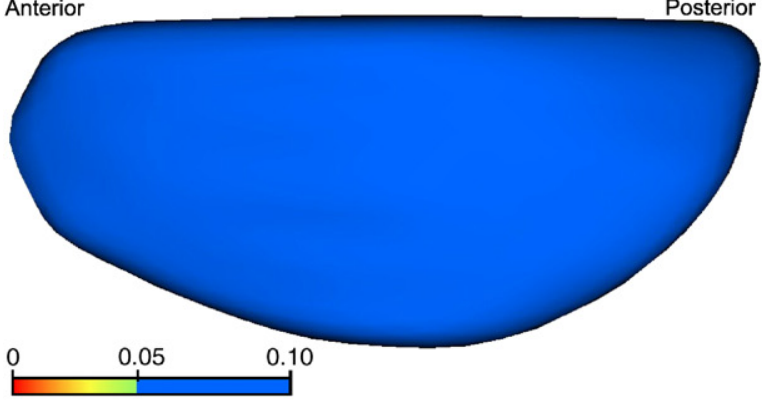

Left Putamen - Medial View

Posterior

Anterior

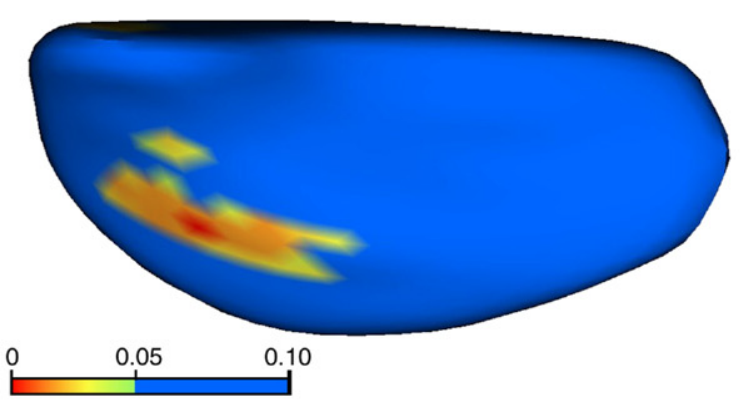

b

Right Putamen - Distance Maps

Right Putamen - Lateral View

Posterior

Anterior

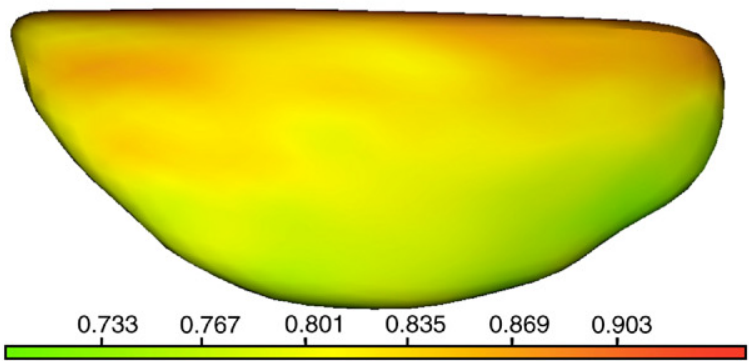

Right Putamen - Medial View

Anterior

Posterior

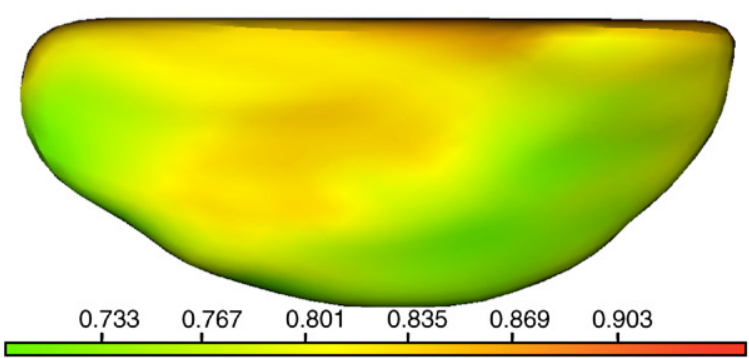

d

Left Putamen - Distance Maps

Left Putamen - Lateral View

Anterior

Posterior

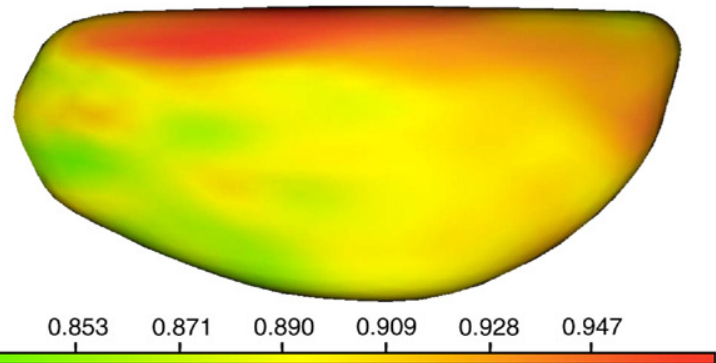

Left Putamen - Medial View

Posterior

Anterior

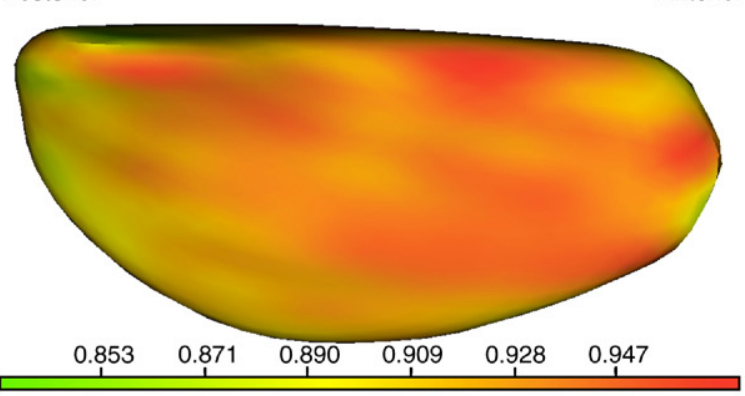

Fig. 6. PNFA versus controls.

located in the dorsal aspect of the striatum concords with previous studies showing a greater dorsal to lesser ventral gradient of atrophy in Huntington's disease (Douaud et al., 2006) and in choreoacanthocytosis (Walterfang et al., 2009), both being genetic neurodegenerative diseases with some clinical features in common with FTLD, and our previous study of the combined FTLD subtypes (Looi et al., 2010). The magnitude of volumetric change is greater posteriorly, indicating an additional posterior-anterior or caudal-rostral gradient, and possibly, a lateral-medial gradient. Parsimoniously, there may be global degeneration in the caudate associated with the clinical features of FTD.

Widespread atrophy in the bilateral caudate in the FTD group compared to controls potentially affects orbito-medial, anterior cingulate and dorsolateral frontostriatal circuits (Haber, 2003; Draganski et al., 2008). The functional organization of frontal cortex and striatal afferent projections is displayed in Fig. 7 (reproduced from Haber, 2003: p.321). The rostro-caudal pattern of connections 
a

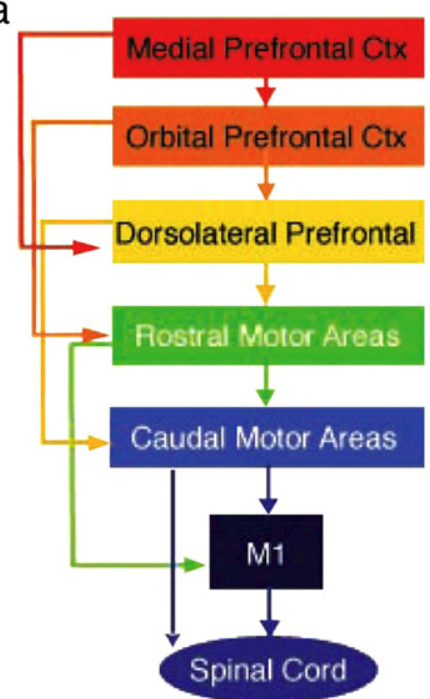

b

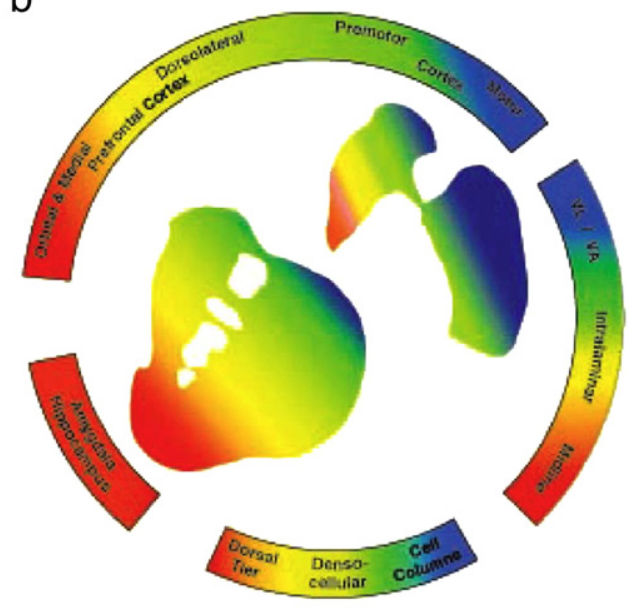

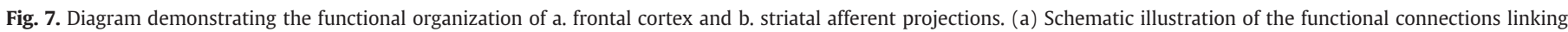

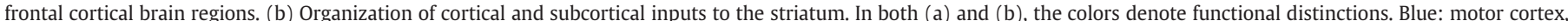

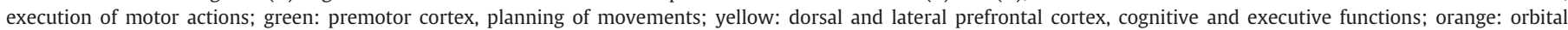

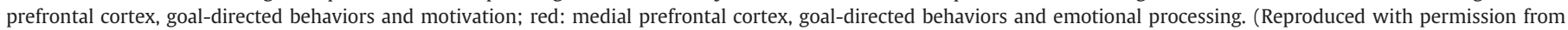
Haber, 2003, fig 3.)

for the caudate shows that the rostral caudate relays orbito-medial fibres; medial rostral aspects relay anterior cingulate fibres; midcaudate relays dorsolateral fibres; whereas the caudal (posterior) aspect relays motor fibres (Draganski et al., 2008). Atrophy in these regions may, by diaschisis, result in the cognitive and behavioral dysfunctions observed in FTD. Since caudate atrophy in FTD is notably posterior, the caudal motoric oculomotor circuit to the frontal eye field may also be significantly compromised, providing as a structural basis for oculomotor function deficits observed in FTLD (Garbutt et al., 2008). Lateral caudate atrophy would significantly impact upon the dorsolateral prefrontal and rostral motor connections.

\subsection{2. $S D$}

There is a very mild relative left-lateralized neuroanatomic basis of differences between SD and controls, in regions linked to medial and orbital prefrontal cortex, and dorsolateral prefrontal cortex. Putative left frontal cortical atrophy associated with this striatal atrophy contrasts with the observed predominant temporal cortical atrophy within SD in this group (Lindberg et al., 2009).

\subsubsection{PNFA}

In PNFA, the atrophic regions of left caudate include the majority of corticostriatal and frontostriatal circuits, similar to that seen bilaterally in FTD. Left-lateralized frontal and temporal lobe atrophy in the PNFA dementia subgroup of this sample, and resultant diaschisis, may partly explain this pattern (Lindberg et al., 2009).

\subsubsection{Overall caudate findings}

The caudate regions affected in FTD impact on interconnections to virtually all frontostriatal circuits, and may thus cause disconnection to all these circuits, potentially resulting in frontal cognitive and oculomotor system dysfunction. In FTD there is a more extensive atrophic process than that the lateralized atrophy seen in PNFA. PNFA subtype classification with phonological paraphasias and agrammatic language may select for patients with left-sided frontostriatal dysfunction, resulting in lateralized atrophy. The relative lack of caudate atrophy in SD is consistent with lesser frontostriatal dysfunction specified in the subtype. Lesser frontostriatal dysfunction, manifest as preserved executive function, is consistent with relative preservation of functional activities of daily living (ADLs) in SD, whereas in FTD, greater frontostriatal dysfunction is associated with impaired ADLs (Mioshi et al., 2007).

\subsection{Putamen}

The rostro-caudal pattern of connections for the putamen shows rostral putamen relays orbito-medial fibres, mid-putamen relays dorsolateral fibres, whilst a larger one-third caudal portion, compared to the caudate, relays motor fibres (Draganski et al., 2008) Thus, the pattern of atrophy in the left putamen in FTD, is in the orbito-medial relay region; with additional dorsolateral and caudal motoric interconnection regional atrophy in the right putamen. Again, this provides support for a potential neuroanatomical basis for corticostriatal motoric dysfunction in FTD. The finding of left putaminal atrophy in FTD in the orbito-medial connectivity region is potentially consistent with involvement of the ventromedial prefrontal cortex which is associated with visceral drives such as eating, sensing internal milieu such as smell, and motivation (Lichter and Cummings, 2001; Mega and Cummings, 2001; Koziol and Budding, 2009); all of which may be affected in FTD.

The motoric linkages of the putamen are to the supplementary motor area and motor cortex (Utter and Basso, 2008), and thus, are corticostriatal circuits. Stereotypies, which are seemingly purposeful repetitive movements found in FTLD, have been found to be associated with striatal atrophy using voxel-based morphometry (Josephs et al., 2008). Those without stereotypies had putaminal atrophy with relative sparing of the caudate (Josephs et al., 2008), indicating that both caudate and putaminal atrophy may be necessary for development of stereotypies in FTD. This supports a specific corticostriatal structural basis for motor dysfunction within the putamen, which, together with caudate atrophy, may result in stereotypies.

The pattern of atrophy in PNFA is very similar to that in FTD, suggesting homogeneity in the neurodegenerative process, whilst SD shows no significant putaminal atrophy.

There is a lesser degree of regional volumetric change in the putamen, indicating that the specific effects on interconnections may be less salient than in the caudate. Anatomically, the caudate has the greater degree of interconnection to frontal regions, and accordingly, the putamen may thus be less affected by frontal cortical atrophy, 
with the resultant putaminal atrophy primarily due to loss of interconnections to parietal motor cortex.

We have demonstrated a potential putaminal basis for both cognitive dysfunction in dorsolateral circuit relays and orbito-medial relays as well as corticostriatal motoric dysfunction (such as stereotypies) in FTD compared to controls, but the displacement maps show that the relative significance and degree of the volumetric change is less than for the caudate. In relation to the putamen there are greater similarities between the pattern of atrophy in FTD and PNFA, than in the caudate, whilst those with SD show no significant atrophy.

\subsection{Summary: Patterns of atrophy}

Our morphologic findings partially support Hypotheses 2 and 3, that there is a gradient in striatal shape consistent with predicted frontostriatal dysfunction, with specific regional atrophy most marked for the caudate, and less so for the putamen. This regional atrophy is significant for the frontostriatal circuits implicated in FTLD, consistent with our previous volumetric findings (Looi et al., 2008b, 2009b), and may arise, in part, from disconnection or diaschisis due to frontal white matter changes (Zhang et al., 2009), as well as from cortical atrophy. In the absence of correlative cortical/subcortical and white matter data, it remains unclear whether the neostriatal changes observed are primary or secondary to atrophy in other regions due to trans-synaptic degeneration. However, we hope to examine such correlations in future studies. This FTLD subtype analysis indicates that the majority of the contribution to the specific patterns of atrophy seen in our previous morphometric comparison of FTLD (all subtypes combined) in comparison to controls (Looi et al., 2010) may be attributed to the FTD, and to a lesser degree, PNFA subtypes. Similarly, the regional neostriatal atrophic basis for motor dysfunction for oculomotor and stereotypic abnormalities in FTLD may arise particularly from the FTD and PNFA subtypes. The SD subtype shows very limited neostriatal atrophy compared to controls.

\subsection{Limitations}

We used a subjective memory complaint cohort for control subjects. However, this group was comprehensively assessed for objective cognitive dysfunction, and those with objective changes were excluded. We acknowledge that the numbers in the subtype groups are relatively small. However, the SPHARM methodology is robust in the analysis of groups of this size, provided there are sufficient comparators, in this case, the controls; but, as the between FTLD subtype numbers were small, regional differences between subtypes were unlikely to be significant after SPHARM FDR correction. Given the exigencies of recruiting people with subtypes of FTLD, AD and controls, age and sex matching were not possible. However, apart from female preponderance, duration of illness and MMSE scores, there were no other significant differences between groups. The subtype showing greatest regional atrophy, FTD, had the shortest average period of disease duration (and smallest range), which would tend to minimize differences in comparison with SD and PNFA [Table 2]. Whilst we did not have neuropathologic confirmation of diagnosis, we had comprehensive clinical assessments for diagnosis consistent with international consensus guidelines on diagnosis.

We acknowledge that manual tracing methods for in vivo neuroanatomy are not necessarily superior to automated image analysis methods. We accept that partial volume effects from the coronal acquisition voxel size of our scans and axial segmentation has the potential to affect our results, limiting sensitivity to change in rostral-ventral regions. Such bias potentially being systematic across all groups, including controls, would likely minimize differences between groups. As a corollary to this argument regarding the plane of manual segmentation, we would argue that segmentation in coronal or sagittal planes would potentially affect sensitivity to change in that plane at the limits of the segmentation, and thus minimize group differences. Whilst automated mapping techniques have the advantage of speed and reliability, there may be issues of validity due to partial volume effects from misclassification of structures and distortion from transformations to templates. Automated algorithms are validated by comparison with manual tracing by experienced tracers. Expert manual segmentation remains the gold standard for in vivo anatomical measurement.

There is a limitation of our shape analyses due to multiple comparisons. Although it is possible to statistically correct for the set of group difference tests of all the subgroups, using a Bonferroni correction, we did not do so within this study as it would result in overly conservative estimates.

\section{Conclusions}

Shape analysis has identified potential neuroanatomical substrates for frontostriatal and corticostriatal circuit dysfunctions in the FTD and PNFA subtypes of FTLD compared to controls.

We have demonstrated the shape of the neostriatum differs between the FTLD subtypes (FTD, PNFA) and controls, partially substantiating hypotheses 1 and 2 (outlined in the Introduction). Lateralization of differences to the left caudate in PNFA may reflect the caudate atrophy relevant to language dysfunction. That the SD subtype shows no significant neostriatal shape change suggests a discontinuity in a structural basis for frontostriatal dysfunction in this group, presumably due to the lesser degree of such dysfunction in the subtype as currently clinically diagnosed. Neostriatal shape change occurs in the gradient FTD $>$ PNFA $>$ SD $>$ Controls, supporting hypothesis 2. Future studies will correlate neuropsychological and clinical measures of dysfunction with MRI morphological analysis of the neostriatum.

In reference to our previous studies of combined FTLD subtype morphology, FTD and PNFA appear to be the main contributors to morphologic change in the broad FTLD group compared to controls, with little significant regional atrophy in SD (Looi et al., 2010). Between the FTLD subtypes, there were few significant differences in morphology, and this may have been due to reduced sensitivity of the analysis to small groups. There was also evidence of general atrophy based in the neostriatum. Future studies should correlate neuropathological findings with MRI morphological analysis of the neostriatum and other frontostriatal circuit components.

Hypothesis 3 was supported in the finding of significant regional atrophy for FTD, and to a lesser extent PNFA, compared to controls, which included most regions specific to frontostriatal circuits in the neostriatum. We also found significant regional atrophy for FTD in the oculomotor regions of the caudate and motoric regions of the putamen, comprising substrates for oculomotor and stereotypic motor dysfunction in FTLD, which, accordingly, may be specific to the FTD subtype. Future studies may examine correlations of observed motor dysfunction in FTLD and MRI morphological analysis.

This study demonstrates the role of differential atrophy in frontostriatal and corticostriatal circuits as potential structural substrates for cognitive and behavioral change in subtypes of FTLD. We suggest longitudinal studies on larger samples, to discern temporal patterns. In this way, we progress, stepwise, towards an understanding of the neurodegenerative process on human brain networks in FTLD; and a spatial-temporal neurodegenerative morphology.

"At first I will tend to think in terms of steps, but nature leaves no gaps, and thus, in the end I will have to see this progression of uninterrupted activity as a whole." (Goethe, 1988, p.75) 


\section{Author contributions}

JCLL designed, coordinated and is guarantor of the study; performed all caudate and putaminal segmentation; and wrote the first draft of the paper. MW conducted the SPHARM-PDM analysis. MS and MN designed the SPHARM-PDM tools and assisted with analysis. LS wrote the software used in volumetric and shape measurement and assisted with binary storage and retrieval. OL assisted with volumetric data extraction and analysis and stereologic intracranial volume measurements. PÖ performed clinical assessments for diagnosis of FTLD subtypes. LB and EÖ assisted with pre-processing of imaging data. PC assisted with inter-rater reliability analysis. DV is the principal investigator working with MW on the SPHARM-PDM analysis. L-OW is the principal investigator. All authors contributed to the editing of the paper.

\section{Acknowledgements}

This research made use of the SMILE medical imaging laboratory at Karolinska University Hospital, Stockholm, Sweden. Christian Andersen, MD, performed neurological assessments for diagnosis of FTLD subtypes. Bram B. Zandbelt assisted with volumetric data extraction and analysis and stereologic intracranial volume measurements. JCLL thanks the Canberra Hospital Specialists Private Practice Trust Fund and ACT Health for partial leave and travel support for the duration of this project, with the remainder of costs self-funded. PÖ's contribution was supported by a grant from the Gun and Bertil Stohne Foundation. We thank all the patients and volunteers.

\section{Appendix A. Computational details of group comparisons, error corrections, and magnitude displacement maps (Reproduced/adapted from Styner et al., 2006)}

\section{Group comparisons of shape}

We calculate group differences by analyzing the spatial location of each point. For this option, no template is necessary and multivariate statistics of the $(x, y, z)$ location is necessary. We have chosen to use the Hotelling $T^{2}$ two sample difference metric as a measurement of how two groups locally differ from each other. The standard Hotelling $T^{2}$ is defined as $T^{2}=(\mu 1-\mu 2)^{\prime}(\Sigma(1 / n 1+1 / n 2))^{-1}(\mu 1-\mu 2)$, where $\Sigma=$ $\left(\Sigma_{1}(n 1-1)+\Sigma_{2}(n 2-1)\right) /(n 1+n 2-2)$ is the pooled covariance matrix. An alternative modified Hotelling $T^{2}$ metric is less sensitive to group differences of the covariance matrixes and the number of samples(Styner et al., 2007): $T^{2}=(\mu 1-\mu 2)^{\prime}\left(\Sigma_{1} 1 / n 1+\Sigma_{2} 1 / n 2\right)^{-1}$ $(\mu 1-\mu 2)$. All our current studies are based on this modified Hotelling $T^{2}$ metric.

We then want to test the two groups for differences in the means of the selected difference metric (univariate: Student $t$, multivariate: Hotelling $T^{2}$ ) at each spatial location. Permutation tests are a valid and tractable approach for such an application, as they rely on minimal assumptions and can be applied even when the assumptions of the parametric approach are untenable. Non-parametric permutation tests are exact, distribution free and adaptive to underlying correlation patterns in the data. Further, they are conceptually straightforward and, with recent improvements in computing power, are computationally tractable.

Our null hypothesis is that the distribution of the locations at each spatial element is the same for every subject regardless of the group. Permutations among the two groups satisfy the exchangeability condition, i.e. they leave the distribution of the statistic of interest unaltered under the null hypothesis. Given $n 1$ members of the first group $a_{k}, k=1 \ldots n 1$ and $n 2$ members of the second group $b_{k}, k=1 \ldots$ $n 2$, we can create $M \leq((n 1+n 2) !) /(n 2$ !) permutation samples. A value of $M$ from 20,000 and up should yield results that are negligibly different from using all permutations.

\section{Corrections for multiple comparisons}

In this study, we are employing non-parametric permutation tests and false discovery rate as two alternative correction methods for the multiple comparison problem.

\section{Correction for Type I errors}

The correction method for multiple comparisons is based on computing first the local $P$-values using permutation tests. The minimum of these $P$-values across the surface is then computed for every permutation. The appropriate corrected $P$-value at level $\alpha$ can then be obtained by the computing the value at the $\alpha$-quantile in the histogram of these minimum values. Using the minimum statistic of the $P$-values, this method correctly controls for the family wise error rate, or the false positives, but no control of the false negatives is provided. The resulting corrected local significance values can thus be regarded as pessimistic estimates akin to a simple Bonferroni correction.

\section{Correction for Type II errors}

Additionally to the non-parametric permutation correction, we have also implemented and applied a False Discovery Rate Estimation (FDR) method. The innovation of this procedure is that it controls the expected proportion of false positives only among those tests for which a local significance has been detected. The FDR method thus allows an expected proportion (usually 5\%) of the FDR corrected significance values to be falsely positive. The correction using FDR provides an interpretable and adaptive criterion with higher power than the non-parametric permutation tests. FDR is further simple to implement and computationally efficient even for large datasets.

The FDR correction is computed as follows:

1. Select the desired FDR bound $q$, e.g. $5 \%$. This is the maximum proportion of false positives among the significant tests that you are willing to tolerate (on average).

2. Sort the $P$-values smallest to largest.

3. Let $p_{q}$ be the $P$-value for the largest index $i$ of the sorted $P$-values $p_{\text {sort,i }} \leq q \cdot i / N$, where $N$ is the number of vertices.

4. Declare all locations with a $P$-value $P \leq p_{\mathrm{q}}$ significant.

\section{Mean difference magnitude difference maps}

These are calculated as the map of the absolute difference in the mean surfaces between groups (based upon the computations above), derived from the lengths of the difference vectors (that is the difference in vectors for analogous surface points between the groups).

\section{References}

Alexander, G.E., Delong, M.R., Strick, P.L., 1986. Parallel organisation of functionally segregated circuits linking basal ganglia and cortex. Annual Review of Neuroscience 9, 357-381.

Bookstein, F.L., 1997. Shape and the information in medical images: a decade of the morphometric synthesis. Computer Vision and Image Understanding 66, 97-118.

Brechbuhler, C., Gerig, G., Kubler, O., 1995. Parametrization of closed surfaces for 3-D shape description. Computer Vision, Graphics, Image Processing 61, 154-170.

Choi, J.-S., Kim, S.H., Yoo, S.Y., Kang, D.-H., Kim, C.-W., Lee, J.-M., Kim, I.Y., Kim, S.I., Kim, Y.Y. Kwon, J.S., 2007. Shape deformity in the corpus striatum in obsessive-compulsive disorder. Psychiatry Research: Neuroimaging 155, 257-264.

Cummings, J.L., 1993. Frontal subcortical circuits and human behaviour. Archives of Neurology 5, 873-880.

Davion, S., Johnson, N., Weintraub, S., Mesulam, M.-M., Engberg, A., Mishra, M., Baker M., Adamson, J., Hutton, R., Rademakers, R., Bigio, E.H., 2007. Clinicopathologic correlations in PGRN mutations. Neurology 69, 1113-1129.

Douaud, G., Gaura, V., Ribeiro, M.-J., Lethimonnier, F., Maroy, R., Verny, C., Krystkowiak, P., Damier, P., Bachoud-Levi, A.-C., Hantraye, P., Remy, P., 2006. Distribution of grey matter atrophy in Huntington's disease: a combined ROI and voxel-based morphometric study. Neuroimage 32, 1562-1575. 
Draganski, B., Kherif, F., Kloppel, S., Cook, P.A., Alexander, D.C., Parker, G.J.M. Deichmann, R., Ashburner, J., Frackowiak, R.S.J., 2008. Evidence for segregated and integrative connectivity patterns in the human basal ganglia. The Journal of Neuroscience 28, 7143-7152.

Garbutt, S., Matlin, A., Helmuth, J., Schenk, A.K., Johnson, J.K., Rosen, H., Dean, D. Kramer, J., Neuhaus, J., Miller, B.L., Lisberger, S.G., Boxer, A.L., 2008. Oculomotor function in frontotemporal lobar degeneration, related disorders and Alzheimer's disease. Brain 131, 1268-1281.

Genovese, C.R., Lazar, N.A., Nichols, T., 2002. Thresholding of statistical maps in functional neuroimaging using the false discovery rate. Neurolmage $15,870-878$

Gerig, G., Styner, M., 2001. Shape versus size: improved understanding of the morphology of brain structures. MICCAI 24-32.

Goethe, J.W.V., 1988. Studies of the physiology of plants. In: Miller, D. (Ed.), Scientific Studies. Suhrkamp, New York, USA.

Haber, S.N., 2003. The primate basal ganglia: parallel and integrative networks. Journal of Chemical Neuroanatomy 26, 317-330.

Haber, S.N., Fudge, J.L., McFarland, N.R., 2000. Striatonigrostriatal pathways in primates form an ascending spiral from the shell to the dorsolateral striatum. The Journal of Neuroscience 20, 2369-2382.

Heimer, L., Van Hoesen, G.W., 2006. The limbic lobe and its output channels: implications for emotional function and adaptive behaviour. Neuroscience and Biobehavioral Reviews 30, 126-147.

Hodges, J.R., Patterson, K., 2007. The neuropsychology of frontotemporal dementia. In: Hodges, J.R. (Ed.), Frontotemporal Dementia Syndromes. Cambridge University Press, Cambridge, UK, pp. 102-133.

Hwang, J., Lyoo, I., Dager, S., Friedman, S., Oh, J., Lee, J., Kim, S., Dunner, D., Renshaw, P. 2006. Basal ganglia shape alterations in bipolar disorder. The American Journal of Psychiatry 163, 276-285.

Josephs, K.A., Whitwell, J.L., Jack, C.R., 2008. Anatomic correlates of stereotypies in frontotemporal lobar degeneration. Neurobiology of Aging 29, 1859-1863.

Koikkalainen, J., Hirvonen, J., Nyman, M., Lötjönen, J., Hietala, J., Ruotsalainen, U., 2007. Shape variability of the human striatum - effects of age and gender. Neuroimage 34, 85-93.

Koziol, L.F., Budding, D.E., 2009. Subcortical Structures and Cognition. Springer, New York.

Leverenz, J.B., Yu, C.E., Montine, T.J., Steinbart, E., Bekris, L.M., Zabetian, C., Kwong, L.K. Lee, V.M.-Y., Schellenberg, G.D., Bird, T.D., 2007. A novel progranulin mutation associated with variable clinical presentation and tau, TDP43 and alpha-synuclein pathology. Brain 130, 1360-1374.

Levitt, J., Westin, C., Nestor, P., Estepar, R., Dickey, C., Voglmaier, M., Seidman, L., Kikinis, R, Jolesz, F., McCarley, R., Shenton, M., 2004. Shape of caudate nucleus and its cognitive correlates in neuroleptic naive schizotypal personality disorder. Biological Psychiatry 55, 177-184.

Levitt, J.J., Styner, M., Niethammer, M., Bouix, S., Koo, M.-S., Voglmaier, M.M., Dickey, C.C. Niznikiewicz, M.A., Kikinis, R., McCarley, R.W., Shenton, M.E., 2009. Shape abnormalities of caudate nucleus in schizotypal personality disorder. Schizophrenia Research $100,127-139$.

Lichter, D.G., Cummings, J.L., 2001. Introduction and overview. In: Lichter, D.G. Cummings, J.L. (Eds.), Frontal-Subcortical Circuits in Psychiatric and Neurological Disorders. Guildford Press, New York, pp. 1-43.

Lindberg, O., Östberg, P., Zandbelt, B.B., Öberg, J., Zhang, Y., Botes, L., Andersen, C., Looi, J.C.L. Bogdanovic, N., Wahlund, L.-O., 2009. Cortical morphometric subclassification of frontotemporal lobar degeneration. AJNR American Journal of Neuroradiology 30, 1233-1239.

Looi, J.C.L., Lindberg, O., Liberg, B, Tatham, V Kumar, R, Maller, J., Millard, E., Sachdev, P., Högberg, G., Pagani, M., Botes, L., Engman, E.-L., Zhang, Y., Svensson, L., Wahlund, L.-O., 2008a. Volumetrics of the caudate nucleus: reliability and validity of a new manual tracing protocol. Psychiatry Research: Neuroimaging 163, 279-288.

Looi, J.C.L., Lindberg, O., Zandbelt, B.B., Östberg, P., Andersen, C., Botes, L., Svensson, L. Wahlund, L.-O., 2008b. Caudate nucleus volume in frontotemporal lobar degeneration - differential atrophy in subtypes of FTLD. AJNR American Journal of Neuroradiology 29, 1537-1543.

Looi, J.C.L., Maller, J., Lindberg, O., Liberg, B., Högberg, G., Pagani, M., Botes, L., Engman, E.-L., Zhang, Y., Svensson, L., Wahlund, L.-O., 2009a. Caudate volumes in public transportation workers exposed to trauma in the Stockholm train system. Psychiatry Research: Neuroimaging 171, 138-143.

Looi, J.C.L., Svensson, L., Lindberg, O., Zandbelt, B.B., Östberg, P., Örndahl, E., Wahlund, L.-O., 2009b. Putaminal volume in frontotemporal lobar degeneration and Alzheimer's Disease - differential volumes in subtypes of FTLD, AD, and controls. AJNR American Journal of Neuroradiology 30, 1552-1560.

Looi, J.C.L., Tatham, V., Kumar, R., Maller, J.J., Millard, E., Wen, W., Brodaty, H., Sachdev, P.S. 2009c. Caudate nucleus volumes in stroke and in vascular dementia. Psychiatry Research: Neuroimaging 174, 67-75.

Looi, J.C.L., Walterfang, M., Styner, M., Svensson, L., Lindberg, O., Östberg, P., Botes, L. Örndahl, E., Chua, P., Kumar, R., Velakoulis, D., Wahlund, L.-O., 2010. Shape analysis of the neostriatum in frontotemporal lobar degeneration, Alzheimer's disease and controls. Neuroimage 51, 970-986.
Lüers, T., Spatz, H., 1957. Picksche Krankheit (Progressive umschriebene Großhirnatrophie.) In: Lubarsch, O., Rössle, F., Henke, F. (Eds.), Handbuch der speziellen pathologischen Anatomie und Histologie, vol.13, part 1A. Springer, Berlin, pp. 614-715.

Mamah, D., Harms, M.P., Wang, L., Barch, D., Thompson, P., Kim, J., Miller, M.I., Csernansky, J.G., 2008. Basal ganglia shape abnormalities in the unaffected siblings of schizophrenia patients. Biological Psychiatry 64, 111-120.

Mega, M., Cummings, J.L., 2001. Frontal subcortical circuits: anatomy and function. In: Salloway, S.P., Malloy, P.F., Duffy, J.D. (Eds.), The Frontal Lobes and Neuropsychiatric Illness. American Psychiatric Press Publishing, Washington DC, pp. 15-32.

Mioshi, E., Kipps, C.M., Dawson, K., Mitchell, J., Graham, A., Hodges, J.R., 2007. Activities of daily living in frontotemporal dementia and Alzheimer disease. Neurology 68, 2077-2084.

Neary, D., Snowden, J.S., Gustafson, L., Passant, U., Stuss, D., Black, S., Freedman, M., Kertesz, A., Robert, P.H., Albert, M., Boone, K., Miller, B.L., Cummings, J., Benson, D.F., 1998. Frontotemporal lobar degeneration: a consensus on clinical diagnostic criteria. Neurology 51, 1546-1554.

Qiu, A., Fennema-Notestine, C., Dale, A.M., Miller, M.I., Alzheimer's Disease Neuroimaging Initiative, 2009. Regional shape abnormalities in mild cognitive impairment and Alzheimer's disease. Neuroimage 45, 656-661.

Rabinovici, G.D., Seeley, W.W., Kim, E.J., Gorno-Tempini, M.L., Rascovsky, K., Pagliaro, T.A., Allison, S.C., Halabi, B.S., Kramer, J.H., Johnson, J.K., Weiner, M.W., Forman, M.S., Trojanowski, J.Q., DeArmond, S.J., Miller, B.L., Rosen, H.J., 2007. Distinct MRI atrophy patterns in autopsy-proven Alzheimer's disease and frontotemporal lobar degeneration. American Journal of Alzheimer's Disease and Other Dementias 22, 474-478.

Schroeter, M.L., Razcka, K., Neumann, J., von Cramon, D.Y., 2007. Towards a nosology for frontotemporal lobar degenerations-a meta-analysis involving 267 subjects. Neuroimage 36, 497-510

Seelaar, H., Schellhaas, H.J., Asmani, A., Kusters, B., Rosso, S., Majoor-Krakauer, D., De Rijk, M.C., Rizzu, P., Brummelhuis, M.T., van Doorn, P.A., Kamphorst, W., Willemsen, R., van Swieten, J.C., 2007. TDP-43 pathology in familial frontotemporal dementia and motor neuron disease without Progranulin mutations. Brain 130, 1375-1385.

Seeley, W.W., Crawford, R., Rascovsky, K., Kramer, J.H., Weiner, M., Miller, B.L., GornoTempini, M.L, 2008. Frontal paralimbic network atrophy in very mild behavioural variant frontotemporal dementia. Archives of Neurology 65, 249-255.

Seeley, W.W., Crawford, R., Zhou, J., Miller, B.L., Greicius, M.D., 2009. Neurodegenerative diseases target large-scale human brain networks. Neuron 62, 42-52.

Styner, M., Lieberman, J., Pantazis, D., Gerig, G., 2004. Boundary and medial shape analysis of the hippocampus in schizophrenia. Medical Image Analysis 8, 197-203.

Styner, M., Lieberman, J.A., McClure, R.K., Weinberger, D.R., Jones, D.W., Gerig, G., 2005. Morphometric analysis of lateral ventricles in schizophrenia and healthy controls regarding genetic and disease-specific factors. Proceedings of the National Academy of Sciences of the United States of America 102, 4872-4877.

Styner, M., Oguz, I., Xu, S., Brechbuhler, C., Pantazis, D., Levitt, J.J., Shenton, M.E., Gerig G., 2006. Framework for the statistical shape analysis of brain structures using SPHARM-PDM. Insight Journal 1-21.

Styner, M., Oguz, I., Xu, S., Pantazis, D., Gerig, G., 2007. Statistical group differences in anatomical shape analysis using the Hotelling T2 metric. Proc SPIE 6512, Medical Imaging 2007, p. 65123. z1-z11.

Utter, A.A., Basso, M.A., 2008. The basal ganglia: an overview of circuits and function. Neuroscience and Biobehavioral Reviews 32, 333-342.

Van Buren, J.M., 1963. Trans-synaptic retrograde degeneration in the visual system of primates. Journal of Neurology, Neurosurgery and Psychiatry 26, 402-409.

von Bagh, K., 1946. Klinische und pathologisch-anatomische Studien an 30 Fällen systematischer umschriebener Atrophie der Grosshirnrinde (Pickscher Krankheit). Annales Academiae Scientiarum Fennicae, Series A. V. Medica - Anthropologica. Suomalaisen Tiedeakatemia: Helsinki.

von Braunmühl, A., 1930. Picksche Krankheit. In: Bumke, O. (Ed.), Handbuch der Geisteskrankheiten, vol. 11, part VII. Springer, Berlin, pp. 673-715.

Walterfang, M., Looi, J.C.L., Danek, A., Walker, R., Bader, B., Evans, A., Mocellin, R., Velakoulis, D., 2009. Size and shape of the neostriatum in neuroacanthocytosis. The Australian and New Zealand Journal of Psychiatry 43 (suppl), A49-A50 (Abstract).

Whitwell, J.L., Jack, C.R., Senjem, M.L., Parisi, J.E., Boeve, B.F., Knopman, D.S., Dickson, D.W., Petersen, R.C., Josephs, K.A., 2009. MRI correlates of protein deposition and disease severity in post-mortem frontotemporal lobar degeneration. Neurodegenerative Diseases 6, 106-117.

Woods, R.P., Grafton, S.T., Holmes, C.J., Cherry, S.R., Mazziotta, J.C., 1998. Automated image registration. I. General methods and intrasubject, intramodality validation. Journal of Computer Assisted Tomography 22, 139-152.

Yucel, Y., Gupta, N., 2008. Glaucoma of the brain: a disease model for the study of transsynaptic neural degeneration. Progress in Brain Research 173, 465-478.

Zhang, Y., Schuff, N., Du, A.T., Rosen, H.J., Kramer, J.H., Gorno-Tempini, M.L., Miller, B.L., Weiner, M.W., 2009. White matter damage in frontotemporal dementia and Alzheimer's disease measured by diffusion MRI. Brain 132, 2579-2592. 